\title{
Stronger Security Notions for Decentralized Traceable Attribute-Based Signatures and More Efficient Constructions
}

\author{
Essam Ghadafi \\ University of Bristol, United Kingdom
}

\begin{abstract}
In this work, we revisit the notion of Decentralized Traceable Attribute-Based Signatures (DTABS) introduced by El Kaafarani et al. (CT-RSA 2014) and improve the state-of-the-art in three dimensions: Firstly, we provide a new stronger security model which circumvents some shortcomings in existing models. Our model minimizes the trust placed in attribute authorities and hence provides, among other things, a stronger definition for non-frameability. In addition, unlike previous models, our model captures the notion of tracing soundness which is important for many applications of the primitive, and which ensures that even if all parties in the system are fully corrupt, no one but the actual signer can claim authorship of the signature. Secondly, we provide a generic construction that is secure w.r.t. our strong security model and show two example instantiations in the standard model which are more efficient than existing constructions (secure under weaker security definitions). Finally, unlike existing constructions, we dispense with the need for the expensive zero-knowledge proofs required for proving tracing correctness by the tracing authority. As a result, tracing a signature in our constructions is significantly more efficient than existing constructions, both in terms of the size of the tracing proof and the computational cost required to generate and verify it. For instance, verifying tracing correctness in our constructions requires only 4 pairings compared to 34 pairings in the most efficient existing construction.
\end{abstract}

Keywords. Attribute-based signatures, security definitions, traceability, standard model.

\section{Introduction}

Attribute-based cryptography provides a versatile solution for designing role-based cryptosystems. In attribute-based cryptosystems, the private operation, e.g. decryption/signing, is performed w.r.t. a security policy. Only users possessing attributes satisfying the policy can perform the operation. Goyal et al. [18], inspired by the work of Sahai and Waters [32], put forward the first attribute-based cryptosystems.

In Attribute-Based Signatures (ABS) [27,28], messages are signed w.r.t. signing policies expressed as predicates. A signature convinces the verifier that it it was produced by a user with attributes satisfying the associated signing policy revealing neither the identity of the user nor the attributes used. Attribute-based signatures have many applications, including trust negotiation, e.g. [12], attribute-based messaging, e.g. [9], and leaking secrets. For more details refer to [28, 31].

The security of attribute-based signatures [27] requires user's privacy and unforgeability. Informally, user's privacy (i.e. anonymity), requires that signatures reveal neither the user's identity nor the attributes used in the signing. On the other hand, unforgeability requires that a user cannot forge a signature w.r.t. a signing predicate that her attributes do not satisfy, even if she colludes with other users.

Traceable Attribute-Based Signatures (TABS) [11] extend standard attribute-based signatures by adding an anonymity revocation mechanism which allows a tracing authority to recover the identity of the signer. Such a feature is important for enforcing accountability and deterring abuse.

Related Work. Various constructions of attribute-based signatures exist in the literature $[26,34,25,28$, $30,31,20,13]$. Those constructions vary in terms of the expressiveness of the policies they support and whether they offer selective or adaptive security. Adaptively secure schemes supporting more expressive policies are preferable since they cover a larger scale of potential applications.

While there exist constructions supporting threshold policies with constant-size signatures, e.g. [20, 13], constructions supporting monotonic/non-monotonic policies, e.g. [28, 30,31], yield signatures that are linearly dependent on the number of attributes in the policy or the systems' security parameter. 
Supporting multiple attribute authorities was first considered by [27, 30]. However, the multi-authority setting still had the problem of requiring a central trusted authority. Furthermore, in some cases, the security of the entire system is compromised if the central authority is corrupted. Okamoto and Takashima [31] proposed the first fully decentralized construction.

Escala et al. [11] added the traceability feature to standard ABS schemes and proposed a model for the single attribute authority setting. More recently, El Kaafarani et al. [10] proposed a security model and two generic constructions for decentralized traceable attribute-based signatures. They also provided instantiations without random oracles [5]. Besides correctness, the recent model of [10] defines three security requirements: anonymity, full unforgeability and traceability. Informally, anonymity requires that a signature reveals neither the identity of the signer nor the set of attributes used in the signing; full unforgeability requires that users cannot forge signatures w.r.t. signing policies their individual attributes do not satisfy even if they collude, which also captures non-frameability; and traceability requires that the tracing authority is always able to establish the identity of the signer and prove such a claim.

We end by noting that there exist other weaker variants of traceable attribute-based signatures suiting specific applications. For instance, [22] proposed the notion of attribute-based group signatures which attaches public attributes to standard group signatures. Also, [23] proposed a traceable attribute-based signature scheme where the signing policy is determined beforehand by the verifier and hence requiring interaction in the signing protocol.

Shortcomings in Existing Models. The unforgeability/non-frameability requirements in all existing models for traceable attribute-based signatures $[11,10]$ (and even those for standard (i.e. non-traceable) attribute-based signatures, e.g. [27,30,31]) besides placing full trust in attribute authorities, assume the existence of secure means for the delivery of the secret attributes' keys from attribute authorities to users. More specifically, learning the key for any attribute a user owns allows framing the user w.r.t. to those attributes. For instance, the non-frameability definition in the single-authority model of [11] relies on the assumption that the attribute authority is fully honest, whereas the full unforgeability definition (also capturing non-frameability) in the stronger and more recent model of [10] assumes that at least one attribute authority is fully honest.

While this is not an issue in standard attribute-based signatures (since signatures are perfectly anonymous and hence it is infeasible for any party to identify the signer), we emphasize that this could be a serious limitation in the traceable setting. In particular, the innocence of users could be jeopardized by being falsely accused of producing signatures they have not produced. A misbehaving attribute authority or any party intercepting the secret attributes' keys is capable of signing on behalf of the user w.r.t. any predicate satisfied by the compromised subset of attributes.

We believe that the overly strong assumptions upon which the unforgeability/non-frameability notions in existing models rely is the result of the absence of the assignment of personal keys to the users. Moreover, the absence of users' personal keys further complicates the constructions and degrades the efficiency. For instance, the recent constructions in [10], similarly to [28], rely on the so-called pseudoattribute technique in order to bind the signature to the message: the user proves that she either owns attributes satisfying the signing predicate or she has a special signature on the message and the encoding of the signing predicate that verifies w.r.t. some trapdoor verification key.

Another shortcoming of existing models for traceable attribute-based signatures is the absence of the tracing soundness requirement which was defined recently in the context of traditional group signatures [33]. This requirement ensures that a valid signature can only trace to a single user even if all entities in the system are fully corrupt. It is vital for many applications, e.g., applications where users get rewarded for signatures they produced or where abusing signing rights might result in legal consequences.

In addition, tracing in existing constructions is costly, both in terms of the size of the tracing proof and the cost for producing and verifying it. The most efficient existing construction [10] requires 34 pairings to verify the opening of a single signature. 
Our Contribution. We first rectify the aforementioned shortcomings in existing models by presenting a stronger security model for the primitive. Our model is for the interesting dynamic and fully decentralized setting in which attributes' management is distributed among different authorities who may not even be aware of one another, and where users and attribute authorities can join the system at any time. Our model offers a stronger security definition for non-frameability which circumvents the limitations inherent in existing models. In addition, our model provides a cleaner definition for traceability, and unlike previous models, it captures the useful notion of tracing soundness [33].

Our second contribution is a generic construction for the primitive which permits expressive signing policies and meets strong adaptive security requirements. Our generic construction dispenses with the expensive zero-knowledge proofs required by existing constructions for proving tracing correctness by deploying a robust, non-interactive tag-based encryption scheme.

Finally, we provide two example instantiations of the generic framework in the standard model. Besides offering stronger security, our instantiations are more efficient than existing constructions. In addition, our constructions have much smaller computation and communication overhead for tracing.

Paper Organization. In Section 2, we give some preliminary definitions. We present our model in Section 3. We list the building blocks we use in Section 4. In Section 5, we present our generic construction and prove its security. In Section 6, we present instantiations in the standard model.

Notation. A function $\nu():. \mathbb{N} \rightarrow \mathbb{R}^{+}$is negligible in $c$ if for every polynomial $p($.$) and all sufficiently$ large values of $c$, it holds that $\nu(c)<\frac{1}{p(c)}$. Given a probability distribution $S$, we denote by $y \leftarrow$ $S$ the operation of selecting an element according to $S$. If $A$ is a probabilistic machine, we denote by $A\left(x_{1}, \ldots, x_{n}\right)$ the output distribution of $A$ on inputs $\left(x_{1}, \ldots, x_{n}\right)$. By PPT we mean running in probabilistic polynomial time in the relevant security parameter.

\section{Preliminaries}

In this section we provide some preliminary definitions.

\subsection{Bilinear Groups}

Let $\mathbb{G}_{1}:=\langle G\rangle, \mathbb{G}_{2}:=\langle\tilde{G}\rangle$ and $\mathbb{G}_{T}$ be groups of a prime order $p$. A bilinear group is a tuple $\mathcal{P}:=$ $\left(\mathbb{G}_{1}, \mathbb{G}_{2}, \mathbb{G}_{T}, p, G, \tilde{G}, e\right)$ where $e: \mathbb{G}_{1} \times \mathbb{G}_{2} \longrightarrow \mathbb{G}_{T}$ is a non-degenerate bilinear map. We will use multiplicative notation for all the groups and let $\mathbb{G}_{1}^{\times}:=\mathbb{G}_{1} \backslash\left\{1_{\mathbb{G}_{1}}\right\}$ and $\mathbb{G}_{2}^{\times}:=\mathbb{G}_{2} \backslash\left\{1_{\mathbb{G}_{2}}\right\}$. We will accent elements from $\mathbb{G}_{2}$ with $\sim$ for the sake of clarity. We use Type-3 groups [14] where $\mathbb{G}_{1} \neq \mathbb{G}_{2}$ and there is no efficient isomorphism between the groups in either direction. We assume the existence of an algorithm BGrpSetup which on input a security parameter $\lambda$ outputs a description of bilinear groups.

\subsection{Complexity Assumptions}

We will use the following assumptions from the literature:

DDH. For a group $\mathbb{G}:=\langle G\rangle$ of a prime order $p$, given $\left(G, G^{a}, G^{b}, C\right) \in \mathbb{G}^{4}$ for $a, b \leftarrow \mathbb{Z}_{p}$, it is hard to decide whether or not $C=G^{a b}$.

SXDH. This assumption requires that the Decisional Diffie-Hellman (DDH) assumption holds in both groups $\mathbb{G}_{1}$ and $\mathbb{G}_{2}$.

$\mathbf{X D L I N}_{\mathbb{G}_{1}}[1]{ }^{1}$. Given $\mathcal{P}$ and the tuple $\left(G^{h}, G^{v}, G^{u}, G^{r h}, G^{s v}, G^{u t}, \tilde{G}^{h}, \tilde{G}^{v}, \tilde{G}^{u}, \tilde{G}^{r h}, \tilde{G}^{s v}\right) \in \mathbb{G}_{1}^{6} \times \mathbb{G}_{2}^{5}$ for unknown $h, r, s, t, u, v \in \mathbb{Z}_{p}$, it is hard to determine whether or not $t=r+s$.

$q$-SDH [8]. Given $\left(G, G^{x}, \ldots, G^{x^{q}}, \tilde{G}, \tilde{G}^{x}\right)$ for $x \leftarrow \mathbb{Z}_{p}$, it is hard to output a pair $\left(c, G^{\frac{1}{x+c}}\right) \in \mathbb{Z}_{p} \times \mathbb{G}_{1}$ for an arbitrary $c \in \mathbb{Z}_{p} \backslash\{-x\}$. 
$q$-AGHO [3]. Given a uniformly random tuple $(G, \tilde{G}, \tilde{W}, \tilde{X}, \tilde{Y}) \in \mathbb{G}_{1} \times \mathbb{G}_{2}^{4}$, and $q$ uniformly random tuples $\left(A_{i}, B_{i}, R_{i}, \tilde{D}_{i}\right) \in \mathbb{G}_{1}^{3} \times \mathbb{G}_{2}$, each satisfying:

$$
\begin{aligned}
& e\left(A_{i}, \tilde{D}_{i}\right)=e(G, \tilde{G}), \\
& e(G, \tilde{X})=e\left(A_{i}, \tilde{W}\right) e\left(B_{i}, \tilde{G}\right) e\left(R_{i}, \tilde{Y}\right),
\end{aligned}
$$

it is hard to output a new tuple $\left(A^{*}, B^{*}, R^{*}, \tilde{D}^{*}\right)$ satisfying the above equations.

\subsection{Span Programs}

For a field $\mathbb{F}$ and a variable set $\mathcal{A}=\left\{\alpha_{1}, \ldots, \alpha_{n}\right\}$, a monotone span program [21] is defined by a $\beta \times \gamma$ matrix $\mathbf{S}$ (over $\mathbb{F}$ ) along with a labeling map $\rho$ which associates each row in $\mathbf{S}$ with an element $\alpha_{i} \in \mathcal{A}$. The span program accepts a set $\mathcal{A}^{\prime}$ iff $\mathbf{1} \in \operatorname{Span}\left(\mathbf{S}_{\mathcal{A}^{\prime}}\right)$, where $\mathbf{S}_{\mathcal{A}^{\prime}}$ is the sub-matrix of $\mathbf{S}$ containing only rows with labels $\alpha_{i} \in \mathcal{A}^{\prime}$, i.e., the program only accepts $\mathcal{A}^{\prime}$ if there exists a vector $\boldsymbol{z}$ s.t. $z \mathbf{S}_{\mathcal{A}^{\prime}}=[1,0, \ldots, 0]$.

\section{Syntax and Security of Decentralized Traceable Attribute-Based Signatures}

A DTABS scheme involves the following entities: a set of attribute authorities, each with a unique identity aid and a pair of secret/verification keys (askaid, avkaid); a tracing authority TA with a secret tracing key tk that is used to identify the signer of a given signature; a set of users, each with a unique identity uid, a personal secret/public key pair (usk[uid], uvk[uid]) and a set of attributes $\mathcal{A} \subseteq \mathbb{A}$ (where $\mathbb{A}$ is the attribute universe). Attributes in the system can be distinctly identified by concatenating the identity of the managing authority with the name of the attribute. This way, the identities (and hence the public keys) of attribute authorities managing attributes appearing in the signing policy can be inferred from the predicate itself which eliminates the need for any additional meta-data to be attached. In our model, attribute authorities as well as users can join the system at any time.

A DTABS scheme is a tuple of polynomial-time algorithms $\mathcal{D} \mathcal{T} \mathcal{A B S}:=($ Setup, AKeyGen, UKeyGen, AttKeyGen, Sign, Verify, Trace, Judge). The definition of the algorithms are as follows; to aid notation all algorithms bar the first three take as implicit input the public parameters pp output by algorithm Setup.

- Setup $\left(1^{\lambda}\right)$ is run by some trusted third party. On input a security parameter $1^{\lambda}$, it outputs public parameters pp and a tracing key tk.

- AKeyGen (pp, aid) is run by attribute authority aid to generate its key pair (ask aid, avk aid). The attribute authority publishes its public key avkaid.

- UKeyGen(pp) outputs a personal secret/verification key pair (usk[uid], uvk[uid]) for the user with identity uid. We assume that the public key table uvk is publicly available (possibly via some PKI) so that anyone can obtain authentic copies of uers' public keys.

- AttKeyGen $\left(\operatorname{ask}_{\operatorname{aid}(\alpha)}\right.$, uid, $\mathbf{u v k [ u i d ] , \alpha )}$ on input the secret key of the attribute authority managing attribute $\alpha$ (i.e. ask aid $(\alpha)$ ), a user's identity uid, a user's personal public key uvk[uid] and an attribute $\alpha \in \mathbb{A}$, it outputs a secret key sk $\mathrm{uid}, \alpha_{\text {for }}$ fortribute $\alpha$ for the user. The key $\mathrm{sk}_{\text {uid, } \alpha}$ is given to uid.

- $\operatorname{Sign}\left(\left\{\operatorname{avk}_{\operatorname{aid}(\alpha)}\right\}_{\alpha \in \mathcal{A}}\right.$, uid, usk[uid], uvk[uid], $\left.\left\{\mathbf{s k}_{\text {uid, } \alpha}\right\}_{\alpha \in \mathcal{A}}, m, \mathbb{P}\right)$ on input an ordered list of attribute authorities' verification keys $\left\{\operatorname{avk}_{\text {aid }(\alpha)}\right\}_{\alpha \in \mathcal{A}}$, a user's identity uid, a user's secret and public keys (usk[uid], uvk[uid]), an ordered list of attributes' secret keys $\left\{\text { sk }_{\text {uid, } \alpha}\right\}_{\alpha \in \mathcal{A}}$ for attributes $\mathcal{A}$ that user uid owns, a message $m$ and a signing predicate $\mathbb{P}$ such that $\mathbb{P}(\mathcal{A})=1$, it outputs a signature $\Sigma$ on $m$ w.r.t. $\mathbb{P}$.

\footnotetext{
${ }^{1}$ It can similarly be defined in $\mathbb{G}_{2}$.
} 
- $\operatorname{Verify}\left(\left\{\operatorname{avk}_{\operatorname{aid}(\alpha)}\right\}_{\alpha \in \mathbb{P}}, m, \Sigma, \mathbb{P}\right)$ on input an ordered list of authorities' verification keys $\left\{\operatorname{avk}_{\text {aid }(\alpha)}\right\}_{\alpha \in \mathbb{P}}$, a message $m$, a signature $\Sigma$ and a predicate $\mathbb{P}$, it verifies whether $\Sigma$ is valid on $m$ w.r.t. $\mathbb{P}$, outputting a bit accordingly.

- Trace(tk, $m, \Sigma, \mathbb{P}, \mathbf{u v k})$ on input the tracing authority's key tk, a message m, a signature $\Sigma$, a signing predicate $\mathbb{P}$, and the public keys table uvk, it outputs an identity uid $>0$ of the signer of $\Sigma$ and a proof $\pi_{\text {Trace }}$ attesting to this claim. If it is unable to trace the signature, it returns $\left(0, \pi_{\text {Trace }}\right)$.

- Judge $\left(\left\{\operatorname{avk}_{\operatorname{aid}(\alpha)}\right\}_{\alpha \in \mathbb{P}}, m, \Sigma, \mathbb{P}\right.$, uid, uvk[uid], $\left.\pi_{\text {Trace }}\right)$ is a deterministic algorithm which on input an ordered list of attribute authorities' verification keys $\left\{\operatorname{avk}_{\text {aid }(\alpha)}\right\}_{\alpha \in \mathbb{P}}$, a message $m$, a signature $\Sigma$, a signing predicate $\mathbb{P}$, a user's identity uid, a user's public verification key uvk[uid], and a tracing proof $\pi_{\text {Trace }}$, it outputs 1 if $\pi_{\text {Trace }}$ is a valid proof that uid has produced $\Sigma$ or 0 otherwise.

\subsection{Security of Decentralized Traceable Attribute-Based Signatures}

The security properties we require from a DTABS scheme are: correctness, anonymity, unforgeability, non-frameability, traceability, and tracing soundness. Unlike the model of El Kaafrani et al. [10], we split the games of unforgeability and non-frameability in order to strengthen the definition of the latter where we allow for the corruption of all authorities. Even though the games of unforgeability and non-frameability could be combined into one game, separating them preserves simplicity. Also, unlike previous models, our model defines the notion of tracing soundness which was recently proposed in the context of group signatures [33].

In our model, we distinguish between bad entities, i.e. those who were initially honest until the adversary learned their secret keys and corrupt entities whose keys have been chosen by the adversary itself.

The experiments used to define the security requirements are shown in Fig. 2. In those experiments, the following global lists are used: HUL is a list of honest users; HAL is a list of honest attribute authorities; HAttL is a list of honestly created users' attributes and has entries of the form (uid, $\alpha$ ); BUL is a list of bad users whose personal secret keys have been revealed to the adversary; BAttL is a list of bad users' attributes whose keys have been revealed to the adversary with entries of the form (uid, $\alpha$ ); BAL is a list of bad attribute authorities whose secret keys have been learned by the adversary; CUL is a list of corrupt users whose keys have been chosen by the adversary; CAL is a list of corrupt attribute authorities whose keys have been chosen by the adversary; SL is a list of signatures obtained from the Sign oracle; $C L$ is a list of challenge signatures obtained from the challenge oracle and is used only in the anonymity game.

The details of the following oracles are given in Fig. 1.

$\operatorname{AddA}($ aid) adds an honest attribute authority with identity aid.

AddU(uid) adds an honest user with identity uid.

$\operatorname{Add} A t t($ uid, $\mathcal{A}$ ) adds honest attributes $\mathcal{A} \subseteq \mathbb{A}$ for user uid. It can be called multiple times to add more attributes for an existing user.

CrptA(aid, vk) adds a corrupt attribute authority whose keys are chosen by the adversary.

$\mathrm{CrptU}$ (uid, vk) adds a corrupt user with identity uid whose personal keys are chosen by the adversary.

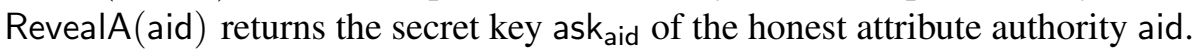

RevealU(uid) returns the personal secret key usk[uid] of user uid.

RevealAtt(uid, $\mathcal{A}$ ) returns the secret keys $\left\{\text { sk }_{\text {uid, } \alpha}\right\}_{\alpha \in \mathcal{A}}$ for attributes $\mathcal{A} \subseteq \mathbb{A}$ owned by user uid. It can be called multiple times.

Sign (uid, $\mathcal{A}, m, \mathbb{P}$ ) returns a signature $\Sigma$ on $m$ using attributes $\mathcal{A}$ belonging to user uid where $\mathbb{P}(\mathcal{A})=1$. $\mathrm{CH}_{b}\left(\left(\right.\right.$ uid $\left._{0}, \mathcal{A}_{0}\right),\left(\right.$ uid $\left.\left._{1}, \mathcal{A}_{1}\right), m, \mathbb{P}\right)$ is a left-right oracle for defining anonymity. On input $\left(\right.$ uid $\left._{0}, \mathcal{A}_{0}\right)$, (uid $\left.{ }_{1}, \mathcal{A}_{1}\right)$, a message $m$ and a signing policy $\mathbb{P}$ with $\mathbb{P}\left(\mathcal{A}_{0}\right)=\mathbb{P}\left(\mathcal{A}_{1}\right)=1$, it returns a signature on $m$ using attributes $\mathcal{A}_{b}$ belonging to user uid ${ }_{b}$ for $b \leftarrow\{0,1\}$.

$\operatorname{Trace}(m, \Sigma, \mathbb{P})$ allows the adversary to ask for signatures to be traced. 


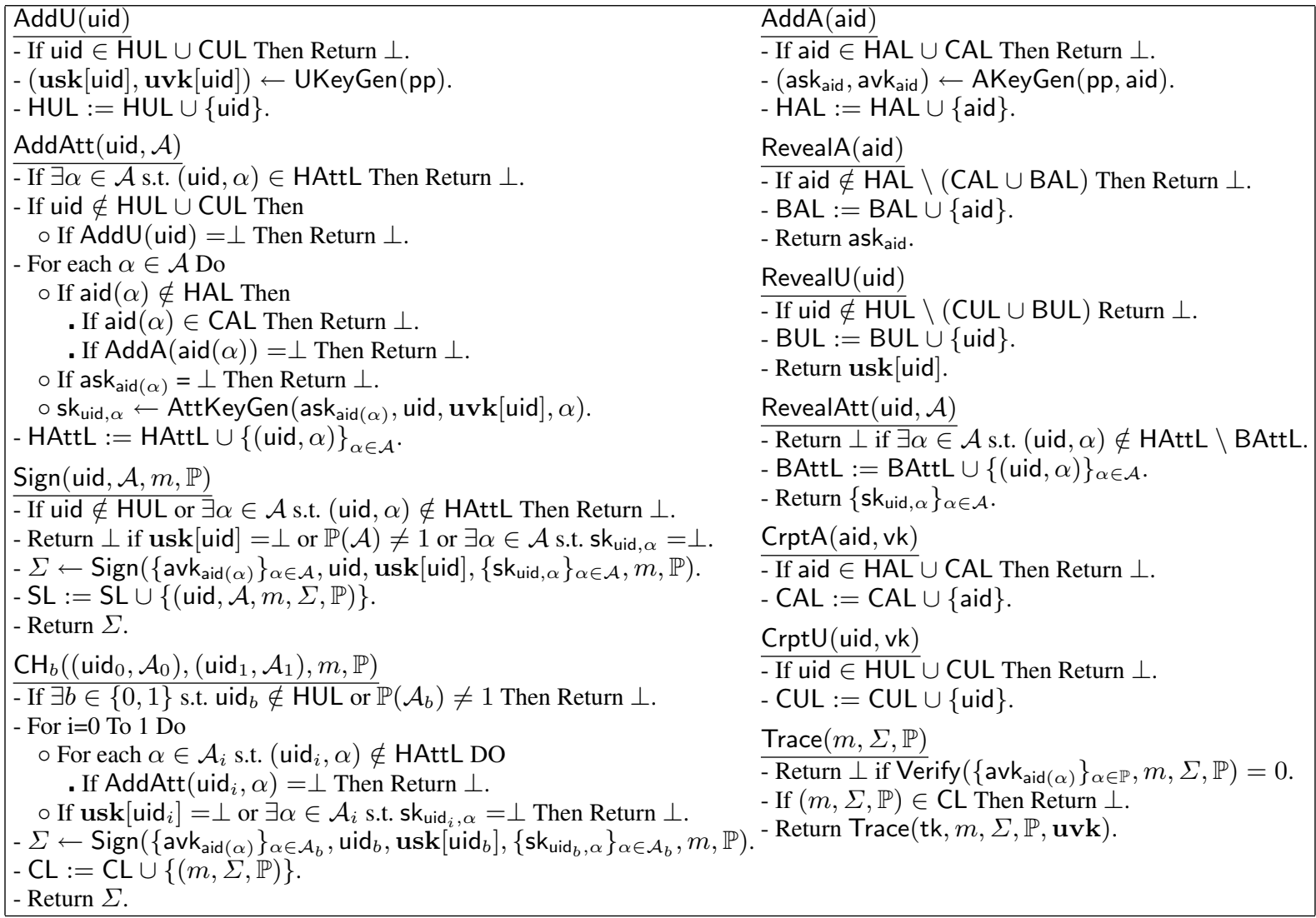

Fig. 1. Oracles used in the security games for DTABS

The details of the security requirements are as follows:

Correctness. This requires that honestly generated signatures verify correctly and trace to the user who produced them. In addition, the Judge algorithm accepts the tracing proof produced by the Trace algorithm. Formally, a DTABS scheme is correct if for all $\lambda \in \mathbb{N}$, all PPT adversaries $\mathcal{B}$ have a negligible advantage $\operatorname{Adv}_{\mathcal{D} \mathcal{T}}^{\text {Corr }} \mathcal{B} \mathcal{S}, \mathcal{B}(\lambda)$ where

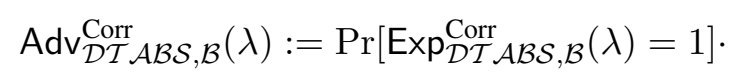

Anonymity. This requires that a signature reveals neither the identity of the user nor the set of attributes used in the signing. In the game, the adversary chooses a message, a signing policy and two users with two, possibly different, sets of attributes satisfying the signing policy. The adversary gets a signature by either user and wins if it correctly guesses the user.

In the game, the adversary can fully corrupt all attribute authorities and learn any user's personal secret key/attribute keys including those used for the challenge. Thus, our definition captures full-key exposure attacks. Since the adversary can sign on behalf of any user, it is redundant to provide it with a sign oracle. The only restriction we impose on the adversary is that it may not query the Trace oracle on the challenge signature.

Our definition captures unlinkability since the adversary has access to all users' personal secret keys/attribute keys.

Formally, a DTABS scheme is (fully) anonymous if for all $\lambda \in \mathbb{N}$, all PPT adversaries $\mathcal{B}$ have a

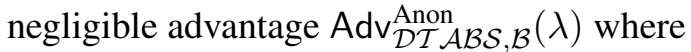

$$
\operatorname{Adv}_{\mathcal{D} \mathcal{T} \mathcal{A B S}, \mathcal{B}}^{\text {Anon }}(\lambda):=\left|\operatorname{Pr}\left[\operatorname{Exp}_{\mathcal{D} \mathcal{T} \mathcal{A} \mathcal{B}, \mathcal{B}}^{\text {Anon-1 }}(\lambda)=1\right]-\operatorname{Pr}\left[\operatorname{Exp}_{\mathcal{D} \mathcal{T} \mathcal{A} \mathcal{B}, \mathcal{B}}^{\text {Anon-0 }}(\lambda)=1\right]\right|
$$




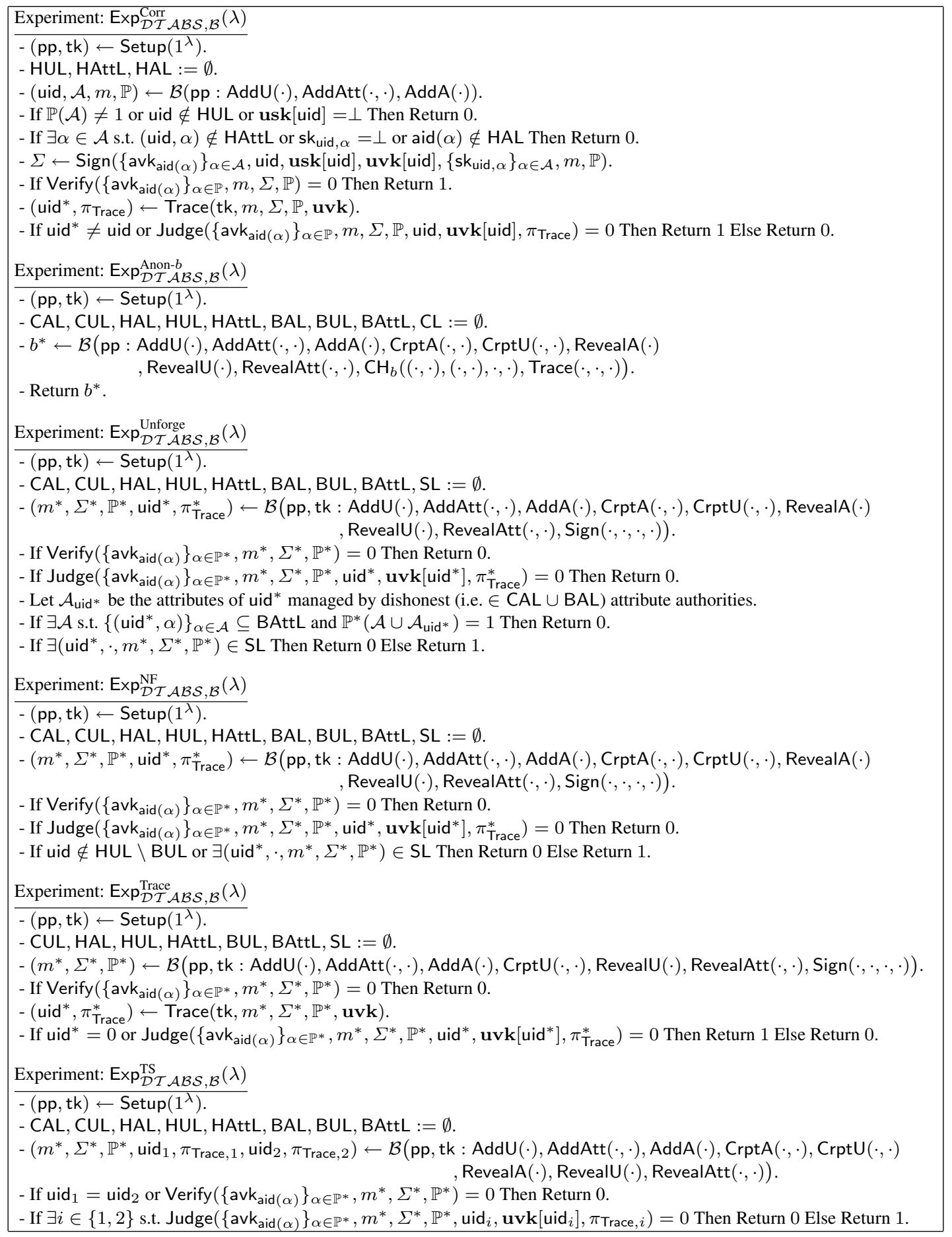

Fig. 2. Security experiments for decentralized traceable attribute-based signatures

Unforgeability. This captures unforgeability scenarios where the forgery opens to a particular user. It guarantees that even if all users in the system pool their individual attributes, they cannot output a signature that traces to a user whose individual attributes do not satisfy the signing predicate. In the game, the adversary can adaptively create corrupt attribute authorities and learn some of the honest authorities' secret keys as long as there is at least a single honest attribute authority managing one of the 
attributes required for satisfying the policy used in the forgery. The adversary can also fully corrupt the tracing authority.

Our definition is adaptive and allows the adversary to adaptively choose both the signing predicate and the message used in the forgery. Note that we consider the stronger variant of unforgeability, i.e. (strong unforgeability) where the adversary wins even if it forges a new signature on a message/predicate pair that was queried to the sign oracle. It is easy to adapt the definition if the weaker variant of unforgeability is desired.

Formally, a DTABS scheme is unforgeable if for all $\lambda \in \mathbb{N}$, all PPT adversaries $\mathcal{B}$ have a negligible advantage $\operatorname{Adv}_{\mathcal{D} \mathcal{T} \mathcal{A} \mathcal{B} \mathcal{S}, \mathcal{B}}^{\text {Unforge }}(\lambda)$ where

$$
\operatorname{Adv}_{\mathcal{D} \mathcal{T} \mathcal{A} \mathcal{B} S, \mathcal{B}}^{\text {Unfore }}(\lambda):=\operatorname{Pr}\left[\operatorname{Exp}_{\mathcal{D} \mathcal{T} \mathcal{A} \mathcal{B} S, \mathcal{B}}^{\text {Unfore }}(\lambda)=1\right]
$$

Non-Frameability. This ensures that even if all authorities and users collude, they cannot produce a signature that traces to an honest user whose personal secret key has not been learned by the adversary.

Our definition guarantees that even if the secret attributes' keys for attributes owned by a user are leaked (for instance, by means of interception or leakage by dishonest attribute authorities), it is still impossible to sign on behalf of the user without knowledge of her personal secret key. Thus, our model overcomes the shortcoming of previous models $[11,10]$ and ensures that an innocent user cannot be framed by dishonest attribute authorities or parties who intercept the communication between the user and the attribute authorities.

In the game, the adversary can fully corrupt all attribute authorities as well as the tracing authority. It can also corrupt as many users of the system as it wishes. We just require that the forgery output by the adversary is a valid signature and traces to a user whose personal secret key has not been revealed to the adversary.

Formally, a DTABS scheme is non-frameable if for all $\lambda \in \mathbb{N}$, all PPT adversaries $\mathcal{B}$ have a negligible advantage $\operatorname{Adv}_{\mathcal{D} \mathcal{T}}^{\mathrm{NF}} \mathcal{B S}, \mathcal{B}(\lambda)$ where

$$
\operatorname{Adv}_{\mathcal{D} \mathcal{T}}^{\mathrm{NF}} \mathcal{A B S}, \mathcal{B}(\lambda):=\operatorname{Pr}\left[\operatorname{Exp}_{\mathcal{D} \mathcal{T}}^{\mathrm{NF}} \mathcal{A B S}, \mathcal{B}(\lambda)=1\right] .
$$

Traceability. This ensures that the adversary cannot produce a signature that cannot be traced. In the game, the adversary is allowed to corrupt the tracing authority and learn both the personal secret key and attributes' keys of any user. However, unlike in the unforgeability and non-frameability games, we require that all the attribute authorities are honest. We emphasize that such an assumption is inevitable as knowing the secret key of any attribute authority would allow the adversary to grant attributes to dummy users resulting in untraceable signature.

Formally, a DTABS scheme is traceable if for all $\lambda \in \mathbb{N}$, all PPT adversaries $\mathcal{B}$ have a negligible advantage $\operatorname{Adv}_{\mathcal{D} \mathcal{T}}^{\operatorname{Trace}} \mathcal{A B}, \mathcal{B}(\lambda)$ where

$$
\operatorname{Adv}_{\mathcal{D} \mathcal{T} \mathcal{A B S}, \mathcal{B}}^{\text {Trace }}(\lambda):=\operatorname{Pr}\left[\operatorname{Exp}_{\mathcal{D} \mathcal{T} \mathcal{A B S}, \mathcal{B}}^{\text {Trace }}(\lambda)=1\right] .
$$

Tracing Soundness. This new requirement, which was not defined in previous models, ensures that even if all authorities (including the tracing authority) and users in the system are all corrupt and collude, they cannot produce a valid signature that traces to two different users. Among other things, this prevents users from claiming authorship of signatures they did not produce or imputing possibly problematic signatures to other users.

Formally, a DTABS scheme satisfies tracing soundness if for all $\lambda \in \mathbb{N}$, all PPT adversaries $\mathcal{B}$ have a negligible advantage $\operatorname{Adv}_{\mathcal{D} \mathcal{T} \mathcal{A B S}, \mathcal{B}}(\lambda)$ where

$$
\operatorname{Adv}_{\mathcal{D} \mathcal{T} \mathcal{A B S}, \mathcal{B}}^{\mathrm{TS}}(\lambda):=\operatorname{Pr}\left[\operatorname{Exp}_{\mathcal{D} \mathcal{T} \mathcal{A B S}, \mathcal{B}}^{\mathrm{TS}}(\lambda)=1\right]
$$




\begin{tabular}{|c|c|}
\hline 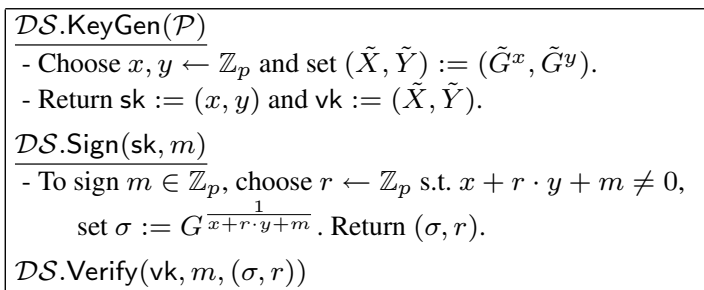 & 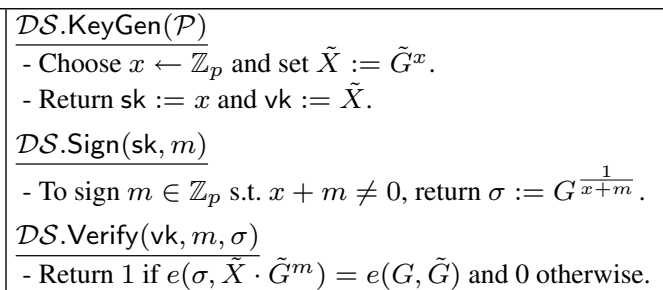 \\
\hline
\end{tabular}

Fig. 3. The full Boneh-Boyen (Left) and the weak Boneh-Boyen (Right) signatures

\section{Building Blocks}

In this section we present the building blocks that we use in our constructions.

\subsection{Digital Signatures}

A digital signature for a message space $\mathcal{M}_{\mathcal{D S}}$ is a tuple of polynomial-time algorithms $\mathcal{D S}:=($ KeyGen, Sign, Verify), where KeyGen outputs a pair of secret/verification keys (sk, vk); Sign(sk, $m$ ) outputs a signature $\sigma$ on the message $m$; Verify $(\mathrm{vk}, m, \sigma)$ outputs 1 if $\sigma$ is a valid signature on $m$.

Existential unforgeability under an adaptive chosen-message attack requires that all PPT adversaries $\mathcal{B}$ have a negligible advantage in the following game:

- A key pair (sk, vk) is generated and $v k$ is given to $\mathcal{B}$.

- Adversary $\mathcal{B}$ makes a polynomial number of queries to a sign oracle Sign(sk, $\cdot)$.

- Eventually, $\mathcal{B}$ halts by outputting $\left(\sigma^{*}, m^{*}\right)$ and wins if $\sigma^{*}$ is valid on $m^{*}$ and $m^{*}$ was never queried to Sign.

A weaker variant of existential unforgeability (i.e. existential unforgeability under a weak chosenmessage attack) requires that the adversary sends all its queries before seeing the verification key. We use two digital signatures by Boneh and Boyen [8], which we refer to as the full (Fig. 3 (Left)) and weak (Fig. 3 (Right)) Boneh-Boyen signature, respectively. Both schemes are secure under the $q$ SDH assumption. The weaker scheme is only secure under a weak chosen-message attack. Let $\mathcal{P}:=$ $\left(\mathbb{G}_{1}, \mathbb{G}_{2}, \mathbb{G}_{T}, p, G, \tilde{G}, e\right)$ be the description of an asymmetric bilinear group. The schemes are given in Fig. 3.

\subsection{Tagged Signatures}

Tagged signatures [10] are digital signatures where the signing and verification algorithms take as an additional input a tag $\tau$. Formally, a tagged signature scheme for a message space $\mathcal{M}_{\mathcal{T} \mathcal{S}}$ and a tag space $\mathcal{T}_{\mathcal{T} \mathcal{S}}$ is a tuple of polynomial-time algorithms $\mathcal{T S}:=\left(\right.$ Setup, KeyGen, Sign, Verify), where Setup $\left(1^{\lambda}\right)$ outputs common public parameters param; KeyGen(param) outputs a pair of secret/verification keys (sk, vk); $\operatorname{Sign}(\mathrm{sk}, \tau, m)$ outputs a signature $\sigma$ on the tag $\tau$ and the message $m$; Verify (vk, $\tau, m, \sigma)$ outputs 1 if $\sigma$ is a valid signature on $\tau$ and $m$. Besides correctness, the security of a tagged signature [10] requires existential unforgeability under an adaptive chosen-message-tag attack which is similar to the definition of existential unforgeability of digital signatures.

We use two instantiations of tagged signatures based on two structure-preserving signature schemes [2] by Abe et al. [3]. The first instantiation (shown in Fig. 4 (Left)) is based on the re-randomizable signature scheme in [3] which signs messages in $\mathbb{G}_{2}^{2}$. We refer to this scheme as AGHO1 after its authors. The tag space of this instantiation is $\mathcal{T}_{\mathcal{T S}}:=\mathbb{G}_{2}$, and the message space is $\mathcal{M}_{\mathcal{T S}}:=\mathbb{G}_{2}$. The tagged signature size is $\mathbb{G}_{1}^{2} \times \mathbb{G}_{2}$ and the signature is fully re-randomizable. The unforgeability of the signature scheme rests on an interactive assumption. See [3] for more details. The second instantiation (shown 


\begin{tabular}{|c|c|}
\hline $\begin{array}{l}\mathcal{T} \mathcal{S} \text {.KeyGen }(\mathcal{P}) \\
-x_{1}, x_{2}, y \leftarrow \mathbb{Z}_{p}, \text { set }\left(X_{1}, X_{2}, \tilde{Y}\right):=\left(G^{x_{1}}, G^{x_{2}}, \tilde{G}^{y}\right) \\
\text { - Return }\left(\text { sk }:=\left(x_{1}, x_{2}, y\right), \mathrm{vk}:=\left(X_{1}, X_{2}, \tilde{Y}\right)\right) \\
\mathcal{T} \mathcal{T} \text { S.Sign }(\mathrm{sk}, \tilde{\tau}, \tilde{M}) \\
\text { - } a \leftarrow \mathbb{Z}_{p}, A:=G^{a}, B:=A^{y}, \tilde{D}:=\left(\tilde{G} \cdot \tilde{\tau}^{-x_{1}} \cdot \tilde{M}^{-x_{2}}\right)^{\frac{1}{a}} \\
\text { - Return } \sigma:=(A, B, \tilde{D}) \text {. } \\
\mathcal{T} \mathcal{S} \text {.Verify }(\mathrm{vk}, \tilde{\tau}, \tilde{M}, \sigma) \\
\text { - Return } 1 \text { if } e(A, \tilde{Y})=e(B, \tilde{G}) \\
\quad \text { and } e(A, \tilde{D}) e\left(X_{1}, \tilde{\tau}\right) e\left(X_{2}, \tilde{M}\right)=e(G, \tilde{G})\end{array}$ & $\begin{array}{l}\mathcal{T} \mathcal{T} \text { S.KeyGen }(\mathcal{P}) \\
-w, x,\left\{y_{i}\right\}_{i=1}^{3} \leftarrow \mathbb{Z}_{p}, \text { set }\left(\tilde{W}, \tilde{X}, \tilde{Y}_{i}\right):=\left(\tilde{G}^{w}, \tilde{G}^{x}, \tilde{G}^{y_{i}}\right) . \\
\text { - Return }\left(\mathrm{sk}:=\left(w, x,\left\{y_{i}\right\}_{i=1}^{3}\right), \mathrm{vk}:=\left(\tilde{W}, \tilde{X},\left\{\tilde{Y}_{i}\right\}_{i=1}^{3}\right)\right) . \\
\mathcal{T} \mathcal{T} . \operatorname{Sign}(\mathrm{sk}, \tau, M) \\
-R \leftarrow \mathbb{G}, a \leftarrow \mathbb{Z}_{p}, A:=G^{a}, \tilde{D}:=\tilde{G}^{\frac{1}{a}}, \\
\quad B:=G^{x-a w} \cdot R^{-y_{1}} \cdot \tau^{-y_{2}} \cdot M^{-y_{3}} . \\
\text { - Return } \sigma:=(A, B, \tilde{D}, R) . \\
\mathcal{T} \mathcal{S} \text {.Verify }(\mathrm{vk}, \tau, M, \sigma) \\
\text { - Return 1 if } e(A, \tilde{D})=e(G, \tilde{G}) \text { and } \\
\quad e(G, \tilde{X})=e(A, \tilde{W}) e(B, \tilde{G}) e\left(R, \tilde{Y}_{1}\right) e\left(\tau, \tilde{Y}_{2}\right) e\left(M, \tilde{Y}_{3}\right) .\end{array}$ \\
\hline
\end{tabular}

Fig. 4. Two instantiations of tagged signatures

in Fig. 4 (Right)) is based on the strongly unforgeable signature scheme from [3] whose unforgeability reduces to the non-interactive $q$-AGHO assumption (cf. Section 2). The message space of the underlying signature scheme is $\mathbb{G}_{1}^{3}$ (where the first element is chosen randomly by the signer), we refer to the underlying scheme as AGHO2. The tag space of this instantiation is $\mathcal{T}_{\mathcal{T} \mathcal{S}}:=\mathbb{G}_{1}$, and the message space is $\mathcal{M}_{\mathcal{T S}}:=\mathbb{G}_{1}$. The signature size is $\mathbb{G}_{1}^{3} \times \mathbb{G}_{2}$. In both instantiations $\mathcal{T} \mathcal{S}$. Setup $\left(1^{\lambda}\right)$ outputs $\mathcal{P}:=\left(\mathbb{G}_{1}, \mathbb{G}_{2}, \mathbb{G}_{T}, p, G, \tilde{G}, e\right)$ which is the description of an asymmetric bilinear group.

\subsection{Strongly Unforgeable One-Time Signatures}

A one-time signature scheme is a signature scheme that is unforgeable against an adversary who makes a single signing query. Strong Unforgeability requires that the adversary cannot even forge a new signature on a message queried the sign oracle on. We will instantiate the one-time signature using the full BonehBoyen signature scheme from Fig. 3.

\subsection{Non-Interactive Zero-Knowledge Proofs}

Let $\mathcal{R}$ be an efficiently computable relation on pairs $(x, w)$, where we call $x$ the statement and $w$ the witness. We define the corresponding language $\mathcal{L}$ as all the statements $x$ in $\mathcal{R}$. A Non-Interactive ZeroKnowledge (NIZK) proof system [7] for $\mathcal{R}$ is defined by a tuple of algorithms $\mathcal{N} \mathcal{I Z K}:=$ (Setup, Prove, Verify, Extract, SimSetup, SimProve).

Setup takes as input a security parameter $1^{\lambda}$ and outputs a common reference string crs and an extraction key $\mathrm{xk}$ which allows for witness extraction. Prove takes as input (crs, $x, w)$ and outputs a proof $\pi$ that $(x, w) \in \mathcal{R}$. Verify takes as input (crs, $x, \pi)$ and outputs 1 if the proof is valid, or 0 otherwise. Extract takes as input (crs, $\mathrm{xk}, x, \pi)$ and outputs a witness. SimSetup takes as input a security parameter $1^{\lambda}$ and outputs a simulated reference string $\mathrm{crs}_{\mathrm{Sim}}$ and a trapdoor key tr that allows for proof simulation. SimProve takes as input ( $\left.\operatorname{crs}_{\operatorname{Sim}}, \operatorname{tr}, x\right)$ and outputs a simulated proof $\pi_{\text {Sim }}$ without a witness.

We require: completeness, soundness and zero-knowledge. Completeness requires that honestly generated proofs are accepted; Soundness requires that it is infeasible (but for a small probability) to produce a convincing proof for a false statement; Zero-knowledge requires that a proof reveals no information about the witness used. The formal definitions can be found in Appendix A.

Groth-Sahai Proofs. Groth-Sahai (GS) proofs [19] are efficient non-interactive proofs in the Common Reference String (CRS) model. In this paper, we will be using the SXDH-based instantiation, which is the most efficient instantiation of the proofs [17]. The language for the system has the form $\mathcal{L}:=\left\{\right.$ statement $\mid \exists$ witness : $E_{1}$ (statement, witness) $, \ldots, E_{n}$ (statement, witness) hold $\}$, where $E_{i}$ (statement, $\cdot$ ) is one of the types of equation summarized in Fig. 5, where $X_{1}, \ldots, X_{m} \in \mathbb{G}_{1}$, $\tilde{Y}_{1}, \ldots, \tilde{Y}_{n} \in \mathbb{G}_{2}, x_{1}, \ldots, x_{m}, \tilde{y}_{1}, \ldots, \tilde{y}_{n} \in \mathbb{Z}_{p}$ are secret variables (hence underlined), whereas $A_{i}, T \in$ $\mathbb{G}_{1}, \tilde{B}_{i}, \tilde{T} \in \mathbb{G}_{2}, a_{i}, \tilde{b}_{i}, k_{i, j}, t \in \mathbb{Z}_{p}, t_{T} \in \mathbb{G}_{T}$ are public constants. For clarity, we also accent exponents 


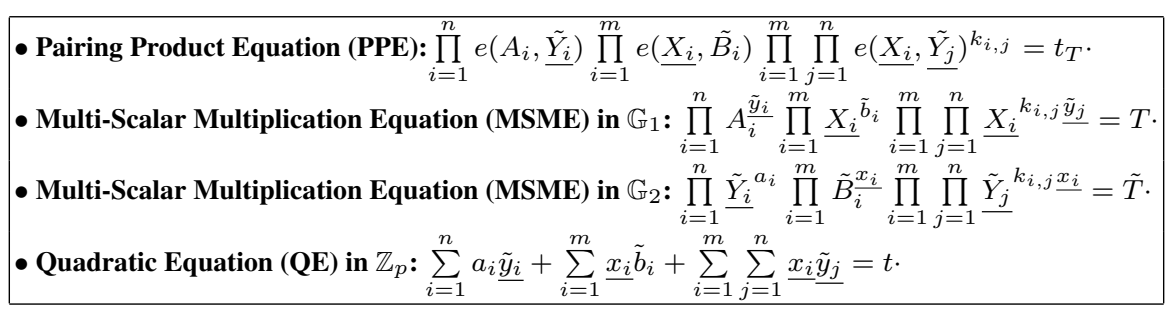

Fig. 5. Types of equations over bilinear groups

to be mapped to group $\mathbb{G}_{2}$ with . The system works by first committing to the elements of the witness and then proving that the commitments satisfy the source equations.

The proof system has perfect completeness, (perfect) soundness, composable witness-indistinguishability/zero-knowledge. Refer to [19] for the formal definitions and details of the instantiations.

\subsection{Robust Non-Interactive Distributed/Threshold Tag-Based Encryption}

In distributed tag-based encryption $[4,15]$, the (tag-based) ciphertexts can only be decrypted if all $n$ decryption servers compute their decryption shares correctly. In the threshold variant, at least $\kappa$ out of $n$ decryption servers must compute their decryption shares correctly for the decryption to succeed. The scheme is non-interactive if decrypting a ciphertext involves no interaction among the decryption servers. The scheme is robust if invalid decryption shares can be identified by the combiner. If the wellformedness of the ciphertext is publicly verifiable, we say the scheme has public verifiability.

Formally, a DTBE scheme for a message space $\mathcal{M}_{\mathcal{D T} \mathcal{T B E}}$ and a tag space $\mathcal{T}_{\mathcal{D T} \mathcal{B E}}$ is a tuple of polynomial-time algorithms (Setup, Enc, IsValid, ShareDec, ShareVerify, Combine), where Setup $\left(1^{\lambda}, n\right)$ outputs a public key and vectors svk $=\left(\mathrm{svk}_{1}, \ldots, \mathrm{svk}_{n}\right)$ and $\mathbf{s k}=\left(\mathrm{sk}_{1}, \ldots, \mathrm{sk}_{n}\right)$ of verification/secret keys for the decryption servers; Enc(pk, $t, m)$ outputs a ciphertext $C_{\mathrm{dtbe}}$ on the message $m$ using tag $t$; Is Valid(pk, $\left.t, C_{\text {dtbe }}\right)$ outputs 1 if the ciphertext is valid under the tag $t$ w.r.t. pk or 0 otherwise; ShareDec(pk, sk $\left.{ }_{i}, t, C_{\mathrm{dtbe}}\right)$ outputs the i-th server decryption share $\nu_{i}$ of $C_{\mathrm{dtbe}}$ or the reject symbol $\perp$; ShareVerify (pk, svk $\left.i, t, C_{\mathrm{dtbe}}, \nu_{i}\right)$ outputs 1 if the decryption share $\nu_{i}$ is valid or 0 otherwise. Combine(pk, $\left.\left\{\mathrm{svk}_{i}\right\}_{i=1}^{n},\left\{\nu_{i}\right\}_{i=1}^{n}, C_{\mathrm{dtbe}}, t\right)$ outputs either the message $m$ or $\perp$.

Besides correctness, we require Selective-Tag weak Indistinguishability against Adaptive Chosen Ciphertext Attacks (ST-wIND-CCA) [24] and Decryption Consistency (DEC-CON). Informally, the former requires that an adversary who gets a decryption oracle for any ciphertext under a tag different from the target tag (which is chosen beforehand), cannot distinguish which challenge message was encrypted. The latter requires that an adversary cannot output two different sets of decryption shares of a ciphertext which open differently. The formal definitions can be found in Appendix B.

For our purpose, it suffices to have a single decryption server, i.e. 1-out-of-1 scheme, therefore the security definitions hereafter are for this setting. We stress, however, that any variant of distributed/threshold tag-based encryption scheme satisfying the properties above can be used in our constructions.

Besides the original scheme given in [15], we also use a second variant of the scheme in [15] (shown in Fig. 6) where we transpose the groups in which the public key and the ciphertext lie. Note that since here we only consider a single decryption server, the verification key svk is redundant as we include all the public elements in the public key pk. Maintaining it is solely for the sake of consistency with the definition of the algorithms.

\section{Our Generic Construction}

In this section, we present our generic construction for decentralized traceable attribute-based signatures. 


\begin{tabular}{|c|c|}
\hline 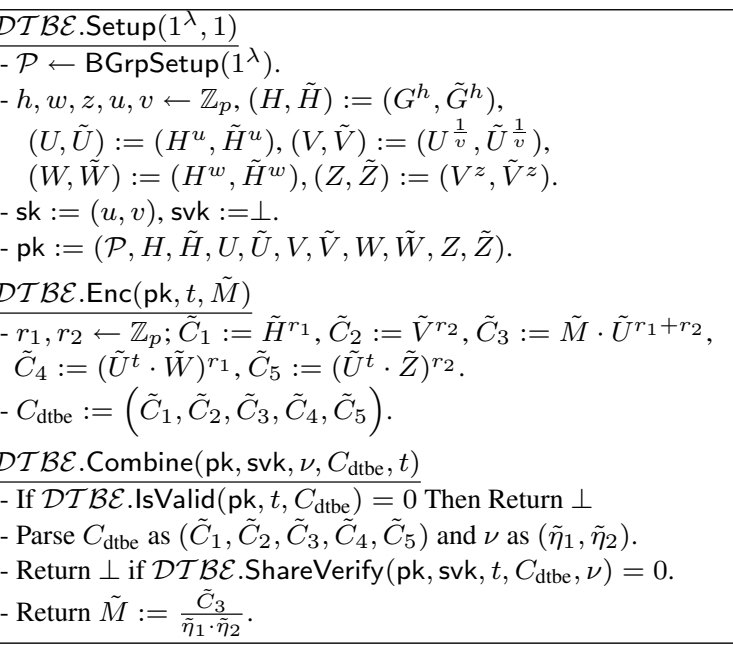 & 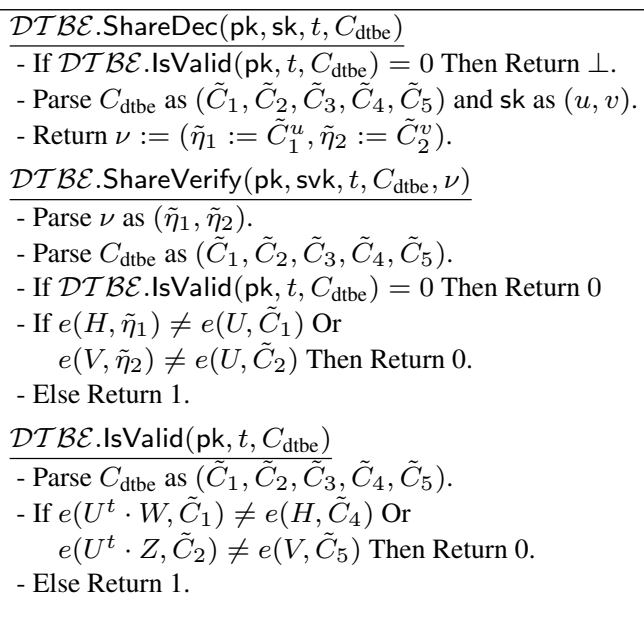 \\
\hline
\end{tabular}

Fig. 6. The transposed 1-out-of-1 variant of the distributed tag-based encryption scheme from [15]

Overview of the construction. Unlike previous constructions, e.g. [10], we dispense with relying on the so-called pseudo-attribute technique to bind the signature to the message and eliminate the need for some of the costly tools required by previous constructions which improves the efficiency of our constructions while offering stronger security than previous ones. Also, we dispense with the need for the expensive zero-knowledge proofs required for proving tracing correctnessns. As a result, tracing (i.e. opening) signatures in our constructions is significantly more efficient than in previous constructions, e.g. [10].

Our construction requires a NIZK proof of knowledge proof system $\mathcal{N} \mathcal{I Z K}$, a selective-tag weakly IND-CCA robust non-interactive (1-out-of-1) distributed tag-based encryption scheme $\mathcal{D} \mathcal{T} \mathcal{B E}$, a tagged signature scheme $\mathcal{T S}$, an existentially unforgeable digital signature scheme $\mathcal{W D S}$ that is secure against a weak chosen-message attack, and a strongly unforgeable one-time signature scheme $\mathcal{O T S}$. Additionally, we require two collision-resistant hash functions $\hat{\mathcal{H}}:\{0,1\}^{*} \rightarrow \mathcal{T}_{\mathcal{D T} \mathcal{B E}}$ and $\mathcal{H}:\{0,1\}^{*} \rightarrow \mathcal{M}_{\mathcal{O T} \mathcal{S}}$. It is sufficient for $\mathcal{W D S}$ to be existentially unforgeable against a weak chosen-message attack as we will use this scheme to sign the verification keys of the one-time signature scheme $\mathcal{O T S}$.

The Setup algorithm generates a common reference string crs for $\mathcal{N} \mathcal{I Z K}$ and runs $\mathcal{D} \mathcal{T} \mathcal{B E}$.Setup to generate the server's secret esk, the verification key esvk and the public key epk. The public parameters of the system is set to $\mathrm{pp}:=\left(1^{\lambda}, \mathrm{crs}\right.$, epk, esvk, $\left.\hat{\mathcal{H}}, \mathcal{H}\right)$. The tracing authority's key is set to tk $:=$ esk.

When a new attribute authority joins the system, it creates a verification/secret key pair ( avk $_{\text {aid }}$, ask ${ }_{\text {aid }}$ ) for the tagged signature scheme $\mathcal{T S}$. When a user joins the system, she generates a verification/secret key pair (uvk[uid], usk[uid]) for the digital signature scheme $\mathcal{W D S}$.

To generate a signing key for attribute $\alpha \in \mathbb{A}$ for user uid, the managing attribute authority signs the user's public key uvk[uid] (used as tag) along with the attribute $\alpha$ using her secret tagged signature signing key. The resulting signature $\sigma_{\alpha}$ is used as the secret key sk $\mathrm{u}_{\text {uid }, \alpha}$ for that attribute by user uid.

To sign a message $m$ w.r.t. a signing policy $\mathbb{P}$, the user chooses a fresh key pair (otsvk, otssk) for the one-time signature $\mathcal{O} \mathcal{T S}$ and encrypts her public key uvk[uid] using the distributed tag-based encryption scheme $\mathcal{D} \mathcal{T} \mathcal{B E}$ (and possibly some randomness $\mu$ ) using $\hat{\mathcal{H}}$ (otsvk) as a tag to obtain a ciphertext $C_{\text {dtbe. }}$ She then signs $\hat{\mathcal{H}}$ (otsvk) using the digital signature scheme $\mathcal{W D S}$ and her personal secret key usk[uid] to obtain a signature $\sigma$. Using $\mathcal{N} \mathcal{I Z K}$, she then computes a proof $\pi$ that: she encrypted her public key correctly, she has a signature $\sigma$ on $\hat{\mathcal{H}}$ (otsvk) that verifies w.r.t. her public key uvk[uid], and she has enough attributes on her public key to satisfy the signing predicate $\mathbb{P}$. To prove the latter, we use a span program (see Section 2.3) represented by the matrix $\mathbf{S}$ : the user proves that she knows a secret vector $\boldsymbol{z} \in \mathbb{Z}_{p}^{|\mathbb{P}|}$ s.t. $\boldsymbol{z} \mathbf{S}=[1,0, \ldots, 0]$. She also needs to show that she possesses a valid tagged signa- 


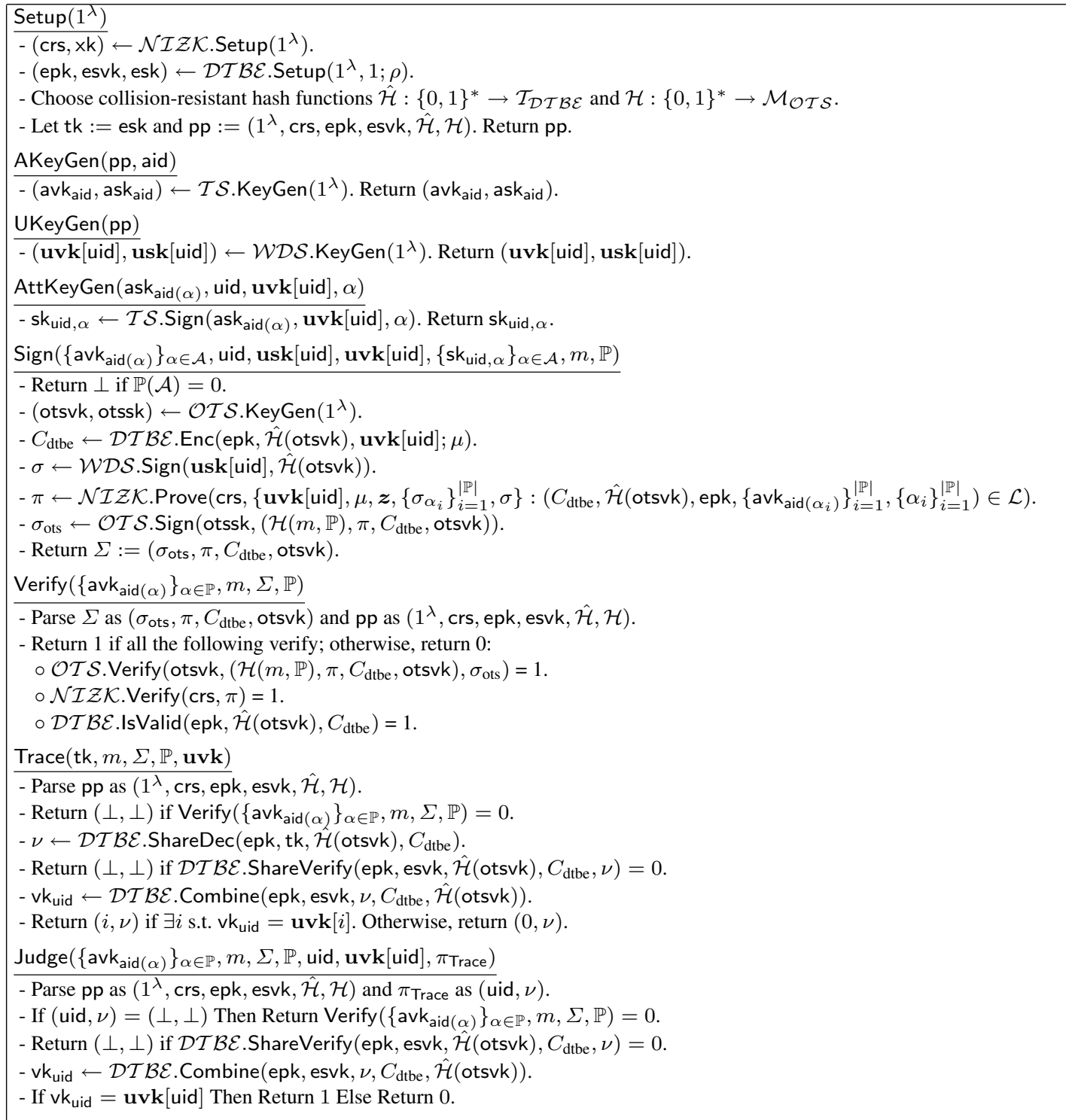

Fig. 7. Our generic construction for DTABS

ture on each attribute in the signing predicate $\mathbb{P}$ for which the corresponding element in $\boldsymbol{z}$ is non-zero. For attributes appearing in $\mathbb{P}$ that the signer does not own, she chooses random signatures. Finally, she signs $\left(\mathcal{H}(m, \mathbb{P}), \pi, C_{\text {dtbe }}\right.$, otsvk) using the one-time signature $\mathcal{O} \mathcal{T} \mathcal{S}$ to obtain a one-time signature $\sigma_{\text {ots }}$. To verify the signature, one just needs to verify the proof $\pi$ and the one-time signature $\sigma_{\text {ots. }}$. We note here that if $\mathcal{T} \mathcal{S}$ and/or $\mathcal{W D S}$ are re-randomizable, one can reveal in the clear the signature components which are independent of uvk[uid] after re-randomizing them. This simplifies the NIZK proof $\pi$ and subsequently improves the efficiency.

To trace a signature, the tracing authority uses its secret key to produce the decryption share $\nu$ of the

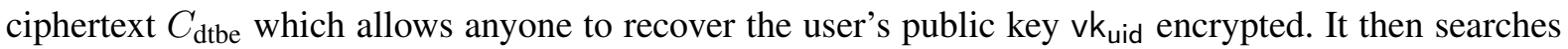
in the public key table uvk to identify the entry matching $v_{\text {uid }}$. It returns (uid, $\nu$ ) if such entry exists, or $(0, \nu)$ otherwise. To verify the tracing correctness, the judge just needs to verify the validity of the decryption share $\nu$ and then recovers the plaintext and verifies that it decrypts to the concerned user. 
The construction is in Fig. 7, whereas the language associated with the NIZK system is as follows, where for clarity we underline the elements of the witness:

$$
\begin{aligned}
& \mathcal{L}:\left\{\left(\left(C_{\text {dtbe }}, \hat{\mathcal{H}} \text { (otsvk) }\right) \text { epk, }\left\{\operatorname{avk}_{\text {aid }\left(\alpha_{i}\right)}\right\}_{i=1}^{|\mathbb{P}|},\left\{\alpha_{i}\right\}_{i=1}^{|\mathbb{P}|}\right),\left(\operatorname{uvk}[\text { uid }], \mu, \boldsymbol{z},\left\{\sigma_{\alpha_{i}}\right\}_{i=1}^{|\mathbb{P}|}\right)\right): \\
& \left(\underline{z} \mathbf{S}=[1,0, \ldots, 0] \bigwedge_{i=1}^{|\mathbb{P}|} \text { if } \underline{z_{i}} \neq 0 \Rightarrow \mathcal{T S} . \operatorname{Verify}\left(\operatorname{avk}_{\operatorname{aid}\left(\alpha_{i}\right)}, \underline{\mathbf{u v k}[\mathrm{uid}]}, \alpha_{i}, \underline{\sigma_{\alpha_{i}}}\right)=1\right)
\end{aligned}
$$

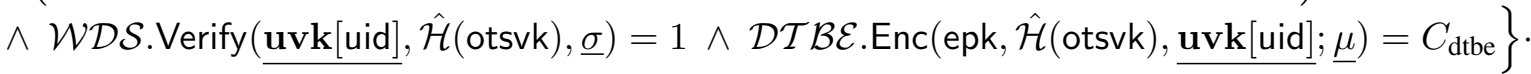

Theorem 1. The construction in Fig. 7 is a secure decentralized traceable attribute-based signature if the building blocks are secure w.r.t. their security requirements.

Next, we present two instantiations of the generic framework in the standard model.

\section{Instantiations in the Standard Model}

\subsection{Instantiation I}

We instantiate $\mathcal{T S}$ using the AGHO1 signature scheme (see Fig. 4 (Left)) and instantiate $\mathcal{W D S}$ and $\mathcal{O} \mathcal{T S}$ using the weak and full Boneh-Boyen signature schemes, respectively. We instantiate $\mathcal{N} \mathcal{I Z K}$ using the Groth-Sahai system, and $\mathcal{D} \mathcal{T} \mathcal{B E}$ using the scheme in Fig. 6.

Let $\mathbf{S} \in \mathbb{Z}_{p}^{|\mathbb{P}|, \beta}$ be the span program for $\mathbb{P}$. To sign, the signer provides the following proofs:

- To prove that $z \mathbf{S}=[1,0, \ldots, 0]$, the signer proves the following linear equations:

$$
\sum_{i=1}^{|\mathbb{P}|}\left(\underline{z}_{i} \tilde{S}_{i, 1}\right)=1 \quad \sum_{i=1}^{|\mathbb{P}|}\left(\underline{z}_{i} \tilde{S}_{i, j}\right)=0, \text { for } j=2, \ldots, \beta
$$

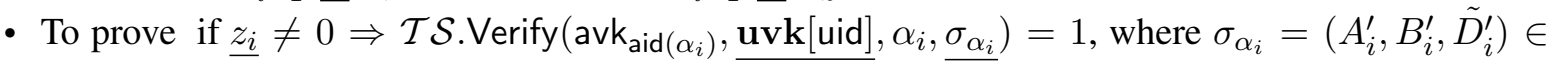
$\mathbb{G}_{1}^{2} \times \mathbb{G}_{2}$ and $\operatorname{avk}_{\text {aid }\left(\alpha_{i}\right)}=\left(X_{i, 1}, X_{i, 2}, \tilde{Y}_{i}\right) \in \overline{\mathbb{G}_{1}^{2} \times \mathbb{G}_{2}}$. The signer re-randomizes $\sigma_{\alpha_{i}}$ by choosing $a^{\prime} \leftarrow \mathbb{Z}_{p}^{*}$ and computing $\sigma_{\alpha_{i}}:=\left(A_{i}, B_{i}, \tilde{D}_{i}\right)=\left(A_{i}^{\prime a^{\prime}}, B_{i}^{\prime a^{\prime}}, \tilde{D}_{i}^{\prime \frac{1}{a^{\prime}}}\right)$, and proves the following

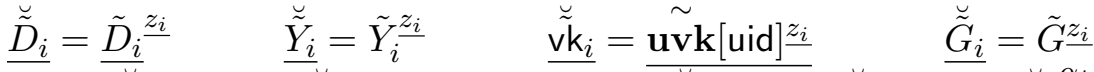

$$
\begin{aligned}
& e\left(A_{i}, \underline{\tilde{\tilde{Y}}_{i}}\right)=e\left(\underline{B_{i}}, \underline{\tilde{\tilde{G}}_{i}}\right) \quad e\left(A_{i}, \underline{\tilde{\tilde{D}}_{i}}\right) e\left(X_{i, 1}, \underline{\tilde{v k}_{i}}\right) e\left(X_{i, 2}, \underline{\tilde{G}}_{i}^{\alpha_{i}}\right)=e\left(G, \underline{\tilde{G}}_{i}\right)
\end{aligned}
$$

Note that the verifier can on her own compute a Groth-Sahai commitment to the value $\breve{\widetilde{G}}_{i}{ }^{\alpha}$ by computing $\mathcal{C}_{\widetilde{G}_{i}}^{\alpha_{i}}$, where $\mathcal{C}_{\breve{G}_{i}}$ is the Groth-Sahai commitment (which is ElGamal ciphertext) to $\breve{\widetilde{G}}_{i}$. Such an observation improves the efficiency. In addition, the way we express the witness of the equations only requires committing to the elements of the vector $z$ in $\mathbb{G}_{1}$, which further improves the efficiency.

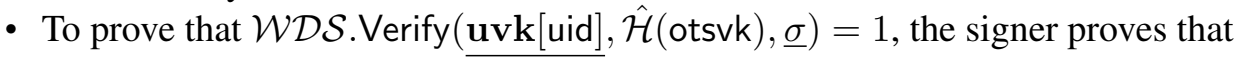

$$
e\left(\underline{\sigma}, \underline{\mathbf{u v k}}[\underline{\text { uid }]}) e\left(\underline{\sigma}, \tilde{G}^{\hat{\mathcal{H}}(\text { otsvk })}\right) e(\underline{G}, \tilde{G})=1 \quad \underline{G}-G=0\right.
$$

- To prove $\mathcal{D} \mathcal{T} \mathcal{B E}$.Enc(epk, $\hat{\mathcal{H}}($ otsvk $\left.), \underline{\mathbf{u v k}[\mathrm{uid}]} ;\left(\underline{r_{1}}, \underline{r_{2}}\right)\right)=C_{\mathrm{dtbe}}$, the signer proves she computed the ciphertext $\left(\tilde{C}_{1}, \tilde{C}_{2}, \tilde{C}_{3}, \tilde{C}_{4}, \tilde{C}_{5}\right)=\left(\tilde{H}^{r_{1}}, \tilde{V}^{r_{2}}, \tilde{U}^{r_{1}+r_{2}} \cdot \tilde{\mathbf{u v k}}[\right.$ uid] $],(\tilde{U} \hat{\mathcal{H}} \text { (otsvk) } \cdot \tilde{W})^{r_{1}},\left(\tilde{U}^{\hat{\mathcal{H}}(\text { otsvk })}\right.$. $\tilde{Z})^{r_{2}}$ ) correctly. It is sufficient to prove that $\tilde{C}_{1}, \tilde{C}_{2}$ and $\tilde{C}_{3}$ were computed correctly and the rest can be verified by checking that $e\left(H, \tilde{C}_{4}\right)=e\left(U^{\hat{\mathcal{H}}(\text { otsvk })} \cdot W, \tilde{C}_{1}\right)$ and $e\left(V, \tilde{C}_{5}\right)=e\left(U^{\hat{\mathcal{H}}(\text { otsvk })} \cdot Z, \tilde{C}_{2}\right)$. Thus, this requires proving $\tilde{C}_{1}=\tilde{H}_{1}^{r_{1}}, \tilde{C}_{2}=\tilde{V}^{r_{2}}$ and $\tilde{C}_{3}=\tilde{U} \underline{r_{1}} \cdot \tilde{U} \underline{r_{2}} \cdot \underline{\mathbf{u v k}}[\mathrm{uid}]$.

The total size of the signature is $\mathbb{G}_{1}^{27 \cdot|\mathbb{P}|+19}+\mathbb{G}_{2}^{22 \cdot|\mathbb{P}|+15}+\mathbb{Z}_{p}^{\beta+3}$. The proof for the following Theorem follows from that of Theorem 1.

Theorem 2. The instantiation is secure if the AGHO1 signature scheme is unforgeable and the assumptions XDLIN $\mathbb{G}_{2}, S X D H$, and $q-S D H$ all hold. 


\begin{tabular}{|c|c|c|c|c|c|c|c|}
\hline Scheme & Signature Size & Model & Setting & No.of.A. & \multicolumn{4}{|c|}{ Tracing } \\
\cline { 6 - 8 } & & & & & Size & Compute & Verify \\
\hline$[11]$ & $\mathbb{G}^{|\mathbb{P}|+\beta+7}$ & ROM & Composite & One & N/A & N/A & N/A \\
{$[10]$} & $\mathbb{G}_{1}^{34 \cdot|\mathbb{P}|+28}+\mathbb{G}_{2}^{32 \cdot|\mathbb{P}|+32}+\mathbb{Z}_{p}^{\beta+1}$ & STD & Prime & Many & $\mathbb{G}_{1}^{3} \times \mathbb{G}_{2}^{4}$ & $4 E_{\mathbb{G}_{1}}+6 E_{\mathbb{G}_{1}}$ & $34 P$ \\
Inst. I & $\mathbb{G}_{1}^{27 \cdot|\mathbb{P}|+19}+\mathbb{G}_{2}^{22 \cdot|\mathbb{P}|+15}+\mathbb{Z}_{p}^{\beta+3}$ & STD & Prime & Many & $\mathbb{G}_{2}^{2}$ & $2 E_{\mathbb{G}_{2}}$ & $4 P$ \\
Inst. II & $\mathbb{G}_{1}^{30 \cdot|\mathbb{P}|+18}+\mathbb{G}_{2}^{30 \cdot|\mathbb{P}|+16}+\mathbb{Z}_{p}^{\beta+3}$ & STD & Prime & Many & $\mathbb{G}_{1}^{2}$ & $2 E_{\mathbb{G}_{1}}$ & $4 P$ \\
\hline
\end{tabular}

Table 1. Efficiency comparison

\subsection{Instantiation II}

To get an efficient instantiation that is based on falsifiable intractability assumptions [29], we instantiate $\mathcal{T S}$ using the AGHO2 signature scheme as shown in Fig. 4 (Right). We needed to transpose the groups from which the public key and the signature components of $\mathcal{W D S}$ are chosen. We also transpose the groups in $\mathcal{D} \mathcal{T} \mathcal{B E}$. The rest of the tools remain the same as in Instantiation I.

Let $\mathbf{S} \in \mathbb{Z}_{p}^{|\mathbb{P}|, \beta}$ be the span program for $\mathbb{P}$. To sign, the signer provides the following proofs:

- To prove that $z \mathbf{S}=[1,0, \ldots, 0]$, the signer proves the following linear equations:

$$
\sum_{i=1}^{|\mathbb{P}|}\left(\tilde{z}_{i} S_{i, 1}\right)=1 \quad \sum_{i=1}^{|\mathbb{P}|}\left(\underline{\tilde{z}}_{i} S_{i, j}\right)=0, \text { for } j=2, \ldots, \beta
$$

- To prove if $\underline{z}_{i} \neq 0 \Rightarrow \mathcal{T} \mathcal{S}$. Verify $\left(\operatorname{avk}_{\operatorname{aid}\left(\alpha_{i}\right)}, \mathbf{u v k}[\right.$ uid] $\left.], \alpha_{i}, \sigma_{\alpha_{i}}\right)=1$, where $\sigma_{\alpha_{i}}=\left(A_{i}, B_{i}, R_{i}, \tilde{D}_{i}\right) \in$ $\mathbb{G}_{1}^{3} \times \mathbb{G}_{2}$ and $\operatorname{avk}_{\text {aid }\left(\alpha_{i}\right)}=\left(\tilde{W}_{i}, \tilde{X}_{i}, \tilde{Y}_{i, 1}, \tilde{Y}_{i, 2}, \overline{\left.Y_{i, 3}\right) \in \mathbb{G}_{2}^{5}}\right.$, the signer proves:

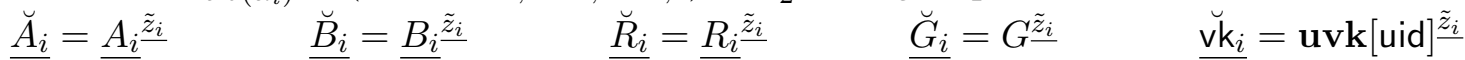

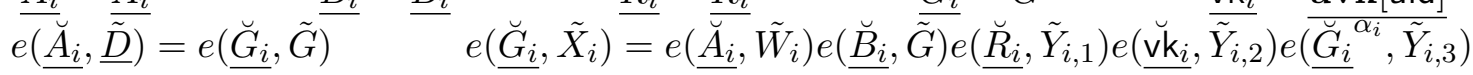

The same two efficiency-enhancing observations used in Instantiation I apply but now in the opposite groups.

- To prove that $\mathcal{W D S}$.Verify $(\mathbf{u v k}[$ uid], $\hat{\mathcal{H}}($ otsvk $), \underline{\tilde{\sigma}})=1$, the signer needs to prove that

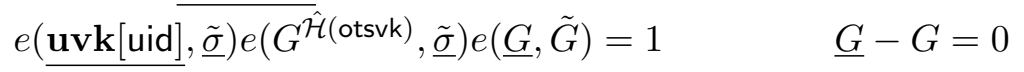

- To prove $\mathcal{D T} \mathcal{B} \mathcal{E}$. Enc(epk, $\hat{\mathcal{H}}($ otsvk $)$, uvk $\left[\right.$ uid] $\left.;\left(\underline{r_{1}}, r_{2}\right)\right)=C_{\text {dtbe }}$, the signer proves she computed

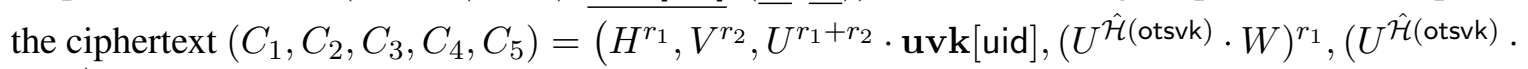
$Z)^{r_{2}}$ ) correctly. It is sufficient to prove that $C_{1}, C_{2}$ and $C_{3}$ were computed correctly and the rest can be verified by checking that $e\left(C_{4}, \tilde{H}\right)=e\left(C_{1}, \tilde{U}^{\hat{\mathcal{H}}(\text { otsvk })} \cdot \tilde{W}\right)$ and $e\left(C_{5}, \tilde{H}\right)=e\left(C_{2}, \tilde{U}^{\hat{\mathcal{H}}(\text { otsvk })} \cdot \tilde{Z}\right)$. Thus, this requires proving $C_{1}=H^{\tilde{r}_{1}}, C_{2}=V^{\frac{\tilde{r}_{2}}{2}}$ and $C_{3}=U \underline{\underline{\tilde{r}_{1}}} \cdot U^{\tilde{\tilde{r}_{2}}} \cdot \mathbf{u v k}[\mathrm{uid}]$.

The total size of the signature is $\mathbb{G}_{1}^{30 \cdot|\mathbb{P}|+18}+\mathbb{G}_{2}^{30 \cdot|\mathbb{P}|+16}+\mathbb{Z}_{p}^{\beta+3}$. The proof for the following Theorem follows from that of Theorem 1 .

Theorem 3. The instantiation is secure if the assumptions XDLIN $\mathbb{G}_{\mathbb{G}_{1}}, q-S D H, q-A G H O$, and SXDH all hold.

We end by noting that in both instantiations signature verification can be mode more efficient by batch verifying GS proofs $[16,6]$.

\subsection{Efficiency Comparison}

We compare the efficiency of our instantiations with that of existing constructions in Table 1. Note here that the construction in [11] is for the single attribute-authority setting and that our constructions are secure w.r.t. a stronger security model than those in $[11,10]$. In the table, $P$ stands for pairing and $E$ is a multi-scalar exponentiation in the group. 


\section{Conclusion}

We have presented a new security model for decentralized traceable attribute-based signatures that is stronger than existing models. In doing so, we have circumvented some shortcomings in existing models. We have also provided a generic framework for obtaining constructions secure w.r.t. our strong model and provided concrete instantiations in the standard model which outperform existing constructions. In addition, tracing signatures in our constructions is much more efficient than previous constructions.

Acknowledgments. The author was supported by ERC Advanced Grant ERC-2010-AdG-267188-CRIPTO and EPSRC via grant EP/H043454/1.

\section{References}

1. M.Abe, M. Chase, B. David, M. Kohlweiss, R. Nishimaki and M. Ohkubo. Constant-Size Structure-Preserving Signatures: Generic Constructions and Simple Assumptions. In ASIACRYPT 2012, Springer LNCS 7658, 4-24, 2012.

2. M. Abe, G. Fuchsbauer, J. Groth, K. Haralambiev and M. Ohkubo. Structure-preserving signatures and commitments to group elements. In CRYPTO 2010, Springer LNCS 6223, 209-236, 2010.

3. M. Abe, J. Groth, K. Haralambiev and M. Ohkubo. Optimal Structure-Preserving Signatures in Asymmetric Bilinear Groups. In CRYPTO 2011, Springer LNCS 6841, 649-666, 2011.

4. S. Arita and K. Tsurudome. Construction of Threshold Public-Key Encryptions through Tag-Based Encryptions. In ACNS 2009, Springer LNCS 5536, 186-200, 2009.

5. M. Bellare and P. Rogaway. Random oracles are practical: A Paradigm for Designing Efficient Protocols. In ACM-CCS 1993, ACM, pp. 62-73.

6. O. Blazy, G. Fuchsbauer, M. Izabach 'ene, A. Jambert, H. Sibert and D. Vergnaud. Batch Groth-Sahai. In ACNS 2010, Springer LNCS 6123, 218-235, 2010.

7. M. Blum, P. Feldman and S. Micali. Non-interactive zero-knowledge and its applications. In STOC 1988, 103-112, 1988.

8. D. Boneh and X. Boyen. Short Signatures Without Random Oracles and the SDH Assumption in Bilinear Groups. In Journal of Cryptology, volume 21(2), 149-177, 2008.

9. R. Bobba, O. Fatemieh, F. Khan, C.A. Gunter and H. Khurana. Using Attribute-Based Access Control to Enable AttributeBased Messaging. In ACSAC 2006, IEEE Computer Society 3027, 403-413, 2006.

10. A. El Kaafarani, E. Ghadafi and D. Khader. Decentralized Traceable Attribute-Based Signatures. In CT-RSA 2014, Springer LNCS 8366, 327-348, 2014.

11. A. Escala, J. Herranz and P. Morillo. Revocable Attribute-Based Signatures with Adaptive Security in the Standard Model. In AFRICACRYPT 2011, Springer LNCS 6737, 224-241, 2011.

12. K.B. Frikken, J. Li and M.J. Atallah. Trust negotiation with hidden credentials, hidden policies, and policy cycles. In NDSS 2006, The Internet Society, 157-172, 2006.

13. Ma. Gagné, S. Narayan and R. Safavi-Naini. Short Pairing-Efficient Threshold-Attribute-Based Signature. In Pairing 2012, Springer LNCS 7708, 295-313, 2012.

14. S. Galbraith, K. Paterson and N.P. Smart. Pairings for cryptographers. Discrete Applied Mathematics, 156, 3113-3121, 2008.

15. E. Ghadafi. Efficient Distributed Tag-Based Encryption and its Application to Group Signatures with Efficient Distributed Traceability. In LATINCRYPT 2014. Springer LNCS 8895, 327-347, 2015.

16. E. Ghadafi, N.P. Smart and B. Warinschi. Practical zero-knowledge proofs for circuit evaluation. In Coding and Cryptography: IMACC 2009, Springer LNCS 5921, 469-494, 2009.

17. E. Ghadafi, N.P. Smart and B. Warinschi. Groth-Sahai proofs revisited. In PKC 2010, Springer LNCS 6056, 177-192, 2010.

18. V. Goyal, O. Pandey, A. Sahai and B. Waters. Attribute-Based Encryption for Fine-Grained Access Control of Encrypted Data. In ACM-CCS 2006, ACM ,89-98, 2006.

19. J. Groth and A. Sahai. Efficient non-interactive proof systems for bilinear groups. In SIAM Journal on Computing, volume 41(5), 1193-1232, 2012.

20. J. Herranz, F. Laguillaumie, B. Libert, and C. Ráfols. Short Attribute-Based Signatures for Threshold Predicates. In CT-RSA 2012, Springer LNCS 7178, 51-67, 2012.

21. M. Karchmer and A. Wigderson. On span programs. In 8th IEEE Structure in Complexity Theory, 102-111, 1993.

22. D. Khader. Attribute Based Group Signatures with Revocation. In Cryptology ePrint Archive, Report 2007/241, http: //eprint.iacr.org/2007/241.pdf.

23. D. Khader, L. Chen, J. H. Davenport. Certificate-Free Attribute Authentication. In Cryptography and Coding: IMACC 2009, Springer LNCS 5921, 301-325, 2009.

24. E. Kiltz. Chosen-Ciphertext Security from Tag-Based Encryption. In TCC 2006, Springer LNCS 3876, 581-600, 2006. 
25. J. Li, M. H. Au, W. Susilo, D. Xie and K. Ren. Attribute-based signature and its applications. In ASIACCS '10, ACM, 60-69, 2010.

26. J. Li and K. Kim. Attribute-Based Ring Signatures. In Cryptology ePrint Archive, Report 2008/394, http: // eprint. iacr.org/2008/394.pdf.

27. H.K. Maji, M. Prabhakaran and M. Rosulek. Attribute-Based Signatures: Achieving Attribute-Privacy and CollusionResistance. In Cryptology ePrint Archive, Report 2008/328, http://eprint.iacr.org/2008/328.pdf.

28. H.K. Maji, M. Prabhakaran and M. Rosulek. Attribute-Based Signatures. In CT-RSA 2011, Springer LNCS 6558, 376392, 2011.

29. M. Naor. On cryptographic assumptions and challenges. In CRYPTO 2003, Springer LNCS 2729, 96-109, 2003.

30. T. Okamoto and K. Takashima. Efficient Attribute-Based Signatures for Non-Monotone Predicates in the Standard Model. In PKC 2011, Springer LNCS 6571, 35-52, ,2011.

31. T. Okamoto and K. Takashima. Decentralized Attribute-Based Signatures. In PKC 2013, Springer LNCS 7778, 125-142, 2012.

32. A. Sahai and B. Waters. Fuzzy Identity-Based Encryption In EUROCRYPT 2005, Springer LNCS 3494, 457-473, 2005.

33. Y. Sakai, J. C. N. Schuldt, K. Emura, G. Hanaoka and K. Ohta. On the Security of Dynamic Group Signatures: Preventing Signature Hijacking. In PKC 2012, Springer LNCS 7293, 715-732, 2012.

34. S. F. Shahandashti and R. Safavi-Naini. Threshold Attribute-Based Signatures and Their Application to Anonymous Credential Systems. In AFRICACRYPT 2009, Springer LNCS 5580, 198-216, 2009.

\section{A Properties of Non-Interactive Zero-Knowledge Proofs}

The properties we require from a non-interctive zero-knowledge proof system are:

- (Perfect) Completeness: $\forall \lambda \in \mathbb{N}, \forall(x, w) \in \mathcal{R}$, we have

$$
\operatorname{Pr}\left[(\operatorname{crs}, \mathbf{x k}) \leftarrow \operatorname{Setup}\left(1^{\lambda}\right) ; \pi \leftarrow \operatorname{Prove}(\operatorname{crs}, x, w): \operatorname{Verify}(\operatorname{crs}, x, \pi)=1\right]=1 .
$$

- Soundness: $\forall \lambda \in \mathbb{N}, \forall x \notin \mathcal{L}$, we have for all adversaries $\mathcal{B}$

$$
\operatorname{Pr}\left[(\operatorname{crs}, \mathrm{xk}) \leftarrow \operatorname{Setup}\left(1^{\lambda}\right) ; \pi \leftarrow \mathcal{B}(\operatorname{crs}, x): \operatorname{Verify}(\operatorname{crs}, x, \pi)=1\right] \leq 2^{-\lambda}
$$

If the above probability is 0 , we say the system has perfect soundness.

- Knowledge Extraction: A proof system is a Proof of Knowledge or has Knowledge Extraction if there exists an efficient extractor algorithm Extract which can extract the witness from any proof the adversary outputs. Note that if a proof system is a proof of knowledge then it is sound. More formally, for all adversaries $\mathcal{B}$, we have

$$
\begin{array}{r}
\operatorname{Pr}\left[(\mathrm{crs}, \mathrm{xk}) \leftarrow \operatorname{Setup}\left(1^{\lambda}\right) ;(x, \pi) \leftarrow \mathcal{B}(\mathrm{crs}) ; w \leftarrow \operatorname{Extract}(\mathrm{crs}, \mathrm{xk}, x, \pi)\right. \\
: \operatorname{Verify}(\mathrm{crs}, x, \pi)=0 \text { OR }(x, w) \in \mathcal{R}] \leq 1-\nu(\lambda) .
\end{array}
$$

If the above probability is 1 , we say the system has perfect knowledge extraction.

- Zero-Knowledge: The system is zero-knowledge if $\forall(x, w) \in \mathcal{R}$, we have for all PPT adversaries $\mathcal{B}$

$$
\begin{aligned}
& \operatorname{Pr}\left[(\operatorname{crs} \operatorname{Sim}, \operatorname{tr}) \leftarrow \operatorname{SimSetup}\left(1^{\lambda}\right): \mathcal{B}^{\operatorname{Sim}\left(\operatorname{crssim}_{\text {Sim }}, \cdot, \cdot\right)}(\operatorname{crs} \operatorname{Sim})=1\right] \\
& \approx \operatorname{Pr}\left[(\operatorname{crs}, \times \mathrm{k}) \leftarrow \operatorname{Setup}\left(1^{\lambda}\right): \mathcal{B}^{\operatorname{Prove}(\operatorname{crs}, \cdot, \cdot)}(\mathrm{crs})=1\right],
\end{aligned}
$$

where $\operatorname{Sim}\left(\operatorname{crs}_{\operatorname{Sim}}, \operatorname{tr}, x, w\right)$ outputs $\operatorname{SimProve}\left(\operatorname{crs}_{\operatorname{Sim}}, \operatorname{tr}, x\right)$ if $(x, w) \in \mathcal{R}$ or $\perp$ otherwise. 


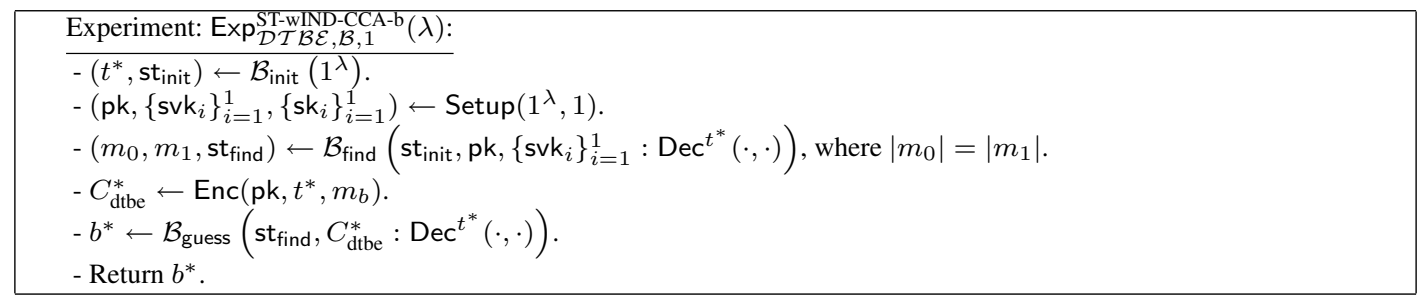

Fig. 8. The ST-wIND-CCA security game for (1-out-of-1) distributed tag-based encryption

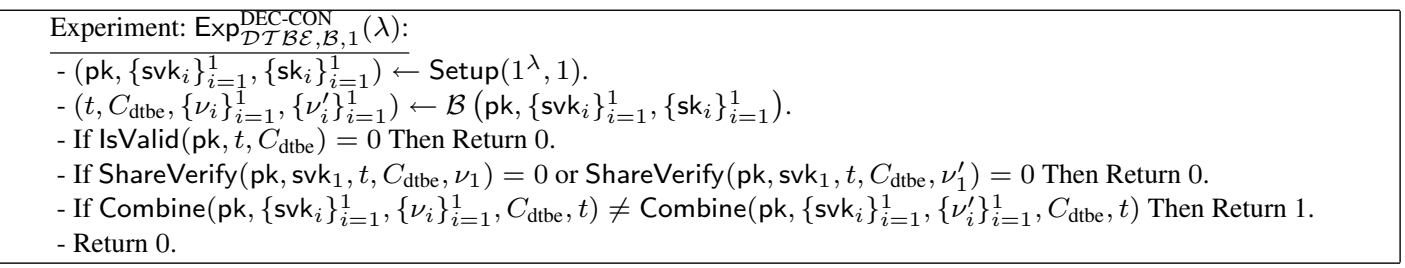

Fig. 9. The decryption consistency game for (1-out-of-1) distributed tag-based encryption

\section{B Security of Robust Non-Interactive Distributed Tag-Based Encryption}

Here we define the security requirements of a (1-out-of-1) distributed tag-based encryption.

- Selective-Tag weak Indistinguishability against Adaptive Chosen Ciphertext Attacks (ST-wINDCCA): This requires that for all $\lambda \in \mathbb{N}$, for all polynomial-time adversaries $\mathcal{B}$ the advantage

$$
\operatorname{Adv}_{\mathcal{D} \mathcal{T}}^{\mathrm{ST}-w \mathcal{B}, \mathcal{B}, 1}(\lambda):=\left|\operatorname{Pr}\left[\operatorname{Exp}_{\mathcal{D} \mathcal{T} \mathcal{B} \mathcal{B}, \mathcal{B}, 1}^{\mathrm{ST}-\mathrm{wIND}-\mathrm{CCA}-1}(\lambda)=1\right]-\operatorname{Pr}\left[\operatorname{Exp}_{\mathcal{D} \mathcal{T} \mathcal{B} \mathcal{B}, \mathcal{B}, 1}^{\mathrm{ST}-\mathrm{CCA}-0}(\lambda)=1\right]\right|
$$

is negligible in $\lambda$, where the game is shown in Fig. 8. In the game, Dec ${ }^{t^{*}}$ rejects any query on $\left(t^{*}, \cdot\right)$ and returns the decryption of the ciphertext otherwise.

- Decryption Consistency (DEC-CON): This requirement [15] requires that for all $\lambda \in \mathbb{N}$, for all PPT adversaries $\mathcal{B}$, the advantage $\operatorname{Adv}_{\mathcal{D} \mathcal{T} \mathcal{B}, \mathcal{B}, 1}^{\mathrm{DEC}-\mathrm{CON}}(\lambda):=\operatorname{Pr}\left[\operatorname{Exp}_{\mathcal{D} \mathcal{T} \mathcal{B} \mathcal{E}, \mathcal{B}, 1}^{\mathrm{DEC}-\mathrm{CON}}(\lambda)=1\right]$ is negligible in $\lambda$, where the game is defined in Fig. 9.

\section{Proof of Theorem 1}

Proof. Correctness of the construction follows from that of the underlying building blocks.

Lemma 1. The construction is non-frameable if $\mathcal{N I Z K}$ is sound, the hash functions $\hat{\mathcal{H}}$ and $\mathcal{H}$ are collision-resistant, and the one-time signature $\mathcal{O} \mathcal{T S}$ and the digital signature $\mathcal{W D S}$ are existentially unforgeable.

Proof. We start by initiating $\mathcal{N} \mathcal{I Z K}$ in the soundness setting which ensures that the adversary cannot break non-frameability by faking proofs for false statements. We show that if there exists an adversary $\mathcal{B}$ that breaks non-frameability, we can construct adversaries: $\mathcal{F}_{1}$ against the unforgeability of the digital signature scheme $\mathcal{W D S}$, adversary $\mathcal{F}_{2}$ against the strong unforgeability of the one-time signature scheme $\mathcal{O} \mathcal{T}$, and adversaries $\mathcal{F}_{3}$ and $\mathcal{F}_{4}$ against the collision-resistance of $\mathcal{H}$ and $\hat{\mathcal{H}}$, respectively, such that

$$
\begin{aligned}
\operatorname{Adv}_{\mathcal{D} \mathcal{T} \mathcal{A B S}, \mathcal{B}}^{\mathrm{NF}}(\lambda) \leq & \kappa(\lambda) \cdot \operatorname{Adv}_{\mathcal{W D S}, \mathcal{F}_{1}}^{\mathrm{Unforg}}(\lambda)+\delta(\lambda) \cdot \operatorname{Adv}_{\mathcal{O} \mathcal{T} \mathcal{S}, \mathcal{F}_{2}}^{\mathrm{Unfore}}(\lambda)+\operatorname{Adv}_{\mathcal{H}, \mathcal{F}_{3}}^{\mathrm{CR}}(\lambda)+ \\
& \operatorname{Adv}_{\hat{\mathcal{H}}, \mathcal{F}_{4}}^{\mathrm{CR}}(\lambda)+\operatorname{Adv}_{\mathcal{N} \mathcal{I} \mathcal{Z} \mathcal{K}, \mathcal{F}_{5}}^{\mathrm{Soud}}(\lambda)
\end{aligned}
$$

where $\kappa(\lambda)$ and $\delta(\lambda)$ are polynomials in $\lambda$ representing an upper bound on the number of honest users and sign queries, respectively, $\mathcal{B}$ is allowed to make in the game. 
The collision-resistance of $\mathcal{H}$ ensures that $\mathcal{B}$ has a negligible probability in finding pairs $\left(m^{*}, \mathbb{P}^{*}\right) \neq$ $(m, \mathbb{P})$ s.t. $\mathcal{H}\left(m^{*}, \mathbb{P}^{*}\right)=\mathcal{H}(m, \mathbb{P})$. If this is not the case, we can use $\mathcal{B}$ to construct an adversary $\mathcal{F}_{3}$ that breaks the collision-resistance of $\mathcal{H}$. Similarly, the collision-resistance of $\hat{\mathcal{H}}$ ensures that $\mathcal{B}$ has a negligible probability in finding two different one-time signature keys otsvk $\neq$ otsvk' s.t. $\hat{\mathcal{H}}($ otsvk $)=$ $\hat{\mathcal{H}}\left(\right.$ otsvk $\left.^{\prime}\right)$. If this is not the case, we can use $\mathcal{B}$ to construct an adversary $\mathcal{F}_{4}$ that breaks the collisionresistance of $\hat{\mathcal{H}}$. Thus, from now on we assume that there are no hash collisions.

- Adversary $\mathcal{F}_{1}$ : Adversary $\mathcal{F}_{1}$ randomly chooses $i \leftarrow\{1, \ldots, \kappa(\lambda)\}$ and guesses that $\mathcal{B}$ will frame user $i$. We have a probability $\frac{1}{\kappa(\lambda)}$ of guessing the correct user. Let $\eta(\lambda)$ denote the number of sign queries by user $i$ adversary $\mathcal{B}$ makes in the game. $\mathcal{F}_{1}$ chooses random key pairs OTSK $:=$ $\left.\{\text { (otsvk, otssk })_{j}\right\}_{j=1}^{\eta(\lambda)}$ for the one-time signature. It forwards $\hat{\mathcal{H}}\left(\right.$ otsvk $\left._{1}\right), \ldots, \hat{\mathcal{H}}\left(\right.$ otsvk $\left._{\eta(\lambda)}\right)$ to its game and gets back a verification key vk and signatures $\sigma_{1}, \ldots, \sigma_{\eta(\lambda)}$. Adversary $\mathcal{F}_{1}$ runs $(\mathrm{crs}, \mathrm{xk}) \leftarrow$ $\mathcal{N} \mathcal{I} \mathcal{K}$. Setup $\left(1^{\lambda}\right)$ and chooses a key tuple (epk, esvk, esk) for $\mathcal{D} \mathcal{T} \mathcal{B E}$. It then forwards pp $:=\left(1^{\lambda}\right.$, crs, epk, esvk, $\mathcal{H}, \hat{\mathcal{H}})$ and tk $:=$ esk to $\mathcal{B}$.

To answer AddA queries, $\mathcal{F}_{1}$ chooses the secret/verification keys for the authority itself so it can answer any AddAtt queries. To answer AddU queries, for all users other than user $i, \mathcal{F}_{1}$ chooses the personal key pair for the user itself. However, for user $i$, it sets its verification key to vk it got from its game (and thus it does not know the corresponding secret key). If in the game $\mathcal{B}$ issues a RevealU query on user $i, \mathcal{F}_{1}$ aborts the game.

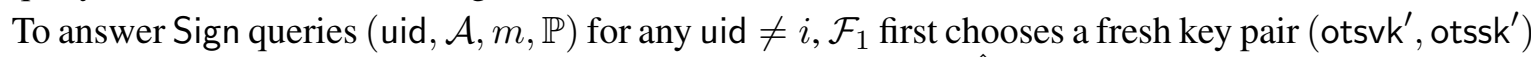
for the one-time signature $\mathcal{O} \mathcal{T}$ and encrypts uvk[uid] using $\hat{\mathcal{H}}$ (otsvk') as a tag and generates the rest of the signature itself. For the $\mathrm{j}$-th sign query by user $i, \mathcal{F}_{1}$ uses the $\mathrm{j}$-th key pair in the set OTSK and encrypts uvk[uid] using $\hat{\mathcal{H}}\left(\mathrm{otsvk}_{j}\right)$ as a tag, and generates the rest of the signature. The rest of $\mathcal{B}$ 's queries are answered normally as in Fig. 1.

Eventually, when $\mathcal{B}$ outputs its forgery, $\mathcal{F}_{1}$ uses the $\mathcal{N} \mathcal{I} \mathcal{Z} \mathcal{K}$ 's extraction key xk to extract the witness and returns the signature $\sigma^{*}$ on $\hat{\mathcal{H}}$ (otsvk ${ }^{*}$ ) that is different from all otsvk ${ }_{1}, \ldots$, otsvk ${ }_{\eta}(\lambda)$ that $\mathcal{F}_{1}$ has used in answering signing queries if $\mathcal{B}$ 's forgery involved framing user $i$ as was guessed by $\mathcal{F}_{1}$. Otherwise, it aborts.

By the existential unforgeability of the signature scheme $\mathcal{W D S}$, the probability of $\mathcal{B}$ winning is negligible.

- Adversary $\mathcal{F}_{2}$ : Adversary $\mathcal{F}_{2}$ gets otsvk* from its game and has access to an oracle Sign that it uses to obtain a single one-time signature that verify w.r.t. otsvk* on a message of its choice. It runs (crs, xk) $\leftarrow \mathcal{N} \mathcal{I} \mathcal{Z} \mathcal{K}$.Setup $\left(1^{\lambda}\right)$ and chooses a key tuple (epk, esvk, esk) for the tag-based encryption scheme $\mathcal{D} \mathcal{T} \mathcal{B E}$. It then forwards pp $:=\left(1^{\lambda}\right.$, crs, epk, esvk, $\left.\mathcal{H}, \hat{\mathcal{H}}\right)$ and tk $:=$ esk to $\mathcal{B}$.

To answer AddA queries, $\mathcal{F}_{2}$ chooses the authority keys itself. To answer AddU queries, $\mathcal{F}_{2}$ chooses the user's key pair itself. To answer AddAtt queries, $\mathcal{F}_{2}$ uses the corresponding authorities' secret keys ask $\mathrm{aid}(\alpha)$ to create the attributes' keys for the user.

Adversary $\mathcal{F}_{2}$ randomly chooses $i \leftarrow\{1, \ldots, \delta(\lambda)\}$ and guesses that $\mathcal{B}$ 's forgery will involve forging a one-time signature that verifies under otsvk* used in answering the i-th signing query.

When asked for the j-th Sign query on (uid, $\mathcal{A}, m, \mathbb{P}$ ), if $j \neq i, \mathcal{F}_{2}$ chooses a fresh key pair (otsvk, otssk) for the one-time signature scheme and answers the query by itself. If $j=i, \mathcal{F}_{2}$ encrypts uvk[uid] using $\hat{\mathcal{H}}$ (otsvk*) (i.e. the verification key it got from its game) as a tag to obtain $C_{\text {dtbe }}$. It then generates $\sigma$ by signing $\hat{\mathcal{H}}$ (otsvk*) using usk[uid] that it chose, and constructs the proof $\pi$. It then forwards $\left(\mathcal{H}(m, \mathbb{P}), \pi, C_{\text {dtbe }}\right.$, otsvk $\left.{ }^{*}\right)$ as the message to its one-time signing oracle to get a one-time signature $\sigma_{\text {ots }} . \mathcal{F}_{2}$ then sends the signature $\Sigma$ to $\mathcal{B}$.

The rest of $\mathcal{B}$ 's queries are answered normally as in Fig. 1.

Eventually, when $\mathcal{B}$ outputs its forgery, $\mathcal{F}_{2}$ aborts if the $\mathcal{B}$ 's forgery did not involve forging a onetime signature that verifies w.r.t. otsvk* it got from its game. The probability that $\mathcal{B}$ forges a one-time signature that verifies w.r.t. otsvk* is $\frac{1}{\delta(\lambda)}$. 
By the strong existential unforgeability of the one-time signature $\mathcal{O} \mathcal{S}, \mathcal{B}$ has a negligible advantage in wining this case.

This concludes the proof.

Lemma 2. The construction is unforgeable if $\mathcal{N} \mathcal{I Z K}$ is sound, the hash functions $\hat{\mathcal{H}}$ and $\mathcal{H}$ are collisionresistant, and the tagged signature $\mathcal{T S}$, and the one-time signature $\mathcal{O} \mathcal{T}$ are existentially unforgeable.

Proof. We show that if there exists an adversary $\mathcal{B}$ breaking unforgeability, we can construct adversaries: $\mathcal{F}_{1}$ against the unforgeability of the tagged signature scheme $\mathcal{T} \mathcal{S}, \mathcal{F}_{2}$ against the strong unforgeability of the one-time signature scheme $\mathcal{O} \mathcal{T} \mathcal{S}$, adversaries $\mathcal{F}_{3}$ and $\mathcal{F}_{4}$ against the collision-resistance of the hash functions $\hat{\mathcal{H}}$ and $\mathcal{H}$, and $\mathcal{F}_{5}$ against the soundness of $\mathcal{N} \mathcal{I Z K}$, such that

$$
\begin{aligned}
\operatorname{Adv}_{\mathcal{D} \mathcal{T} \mathcal{A B S}, \mathcal{B}}^{\text {Unforge }}(\lambda) \leq & \kappa(\lambda) \cdot \operatorname{Adv}_{\mathcal{T} \mathcal{S}, \mathcal{F}_{1}}^{\text {Unforge }}(\lambda)+\delta(\lambda) \cdot \operatorname{Adv}_{\mathcal{O} \mathcal{T} \mathcal{S}, \mathcal{F}_{2}}^{\text {Unforge }}(\lambda)+\operatorname{Adv}_{\hat{\mathcal{H}, \mathcal{F}_{3}}}^{\mathrm{CR}}(\lambda)+ \\
& \operatorname{Adv}_{\mathcal{H}, \mathcal{F}_{4}}^{\mathrm{CR}}(\lambda)+\operatorname{Adv}_{\mathcal{N} \mathcal{I} \mathcal{Z} \mathcal{K}_{,} \mathcal{F}_{5}}^{\text {Sound }}(\lambda),
\end{aligned}
$$

where $\kappa(\lambda)$ and $\delta(\lambda)$ are polynomials in $\lambda$ representing an upper bound on the number of honest attribute authorities and sign queries, respectively, $\mathcal{B}$ is allowed to make in the game.

We instantiate $\mathcal{N} \mathcal{I} \mathcal{Z K}$ in the soundness setting and hence the adversary cannot break unforgeability by faking proofs for false statements. By the collision-resistance of $\hat{\mathcal{H}}, \mathcal{B}$ has a negligible probability in finding two different one-time signature keys otsvk $\neq$ otsvk' $^{\prime}$ s.t. $\hat{\mathcal{H}}\left(\right.$ otsvk $\left.^{\prime}\right)=\hat{\mathcal{H}}$ (otsvk'). If this is not the case, we can use $\mathcal{B}$ to construct an adversary $\mathcal{F}_{3}$ that breaks the collision-resistance of $\hat{\mathcal{H}}$. Similarly, by the security of $\mathcal{H}, \mathcal{B}$ has a negligible probability in finding collisions $(m, \mathbb{P}) \neq\left(m^{*}, \mathbb{P}^{*}\right)$ s.t. $\mathcal{H}(m, \mathbb{P})=\mathcal{H}\left(m^{*}, \mathbb{P}^{*}\right)$. If this is not the case, we can use $\mathcal{B}$ to construct an adversary $\mathcal{F}_{4}$ that breaks the collision-resistance of $\mathcal{H}$. Thus, from now on we assume that there are no hash collisions.

- Adversay $\mathcal{F}_{1}$ : Adversary $\mathcal{F}_{1}$ gets the tagged signature scheme's verification key vk from its game and has access to an oracle Sign that it uses to obtain tagged signatures that verify w.r.t. vk on messages and tags of its choice. Adversary $\mathcal{F}_{1}$ starts by running (crs, xk $) \leftarrow \mathcal{N} \mathcal{I Z K}$. Setup $\left(1^{\lambda}\right)$ and choosing a key tuple (epk, esvk, esk) for the distributed tag-based encryption scheme $\mathcal{D} \mathcal{T} \mathcal{B E}$. It forwards pp $:=\left(1^{\lambda}\right.$, crs, epk, esvk, $\left.\mathcal{H}, \hat{\mathcal{H}}\right)$ and tk $:=$ esk to $\mathcal{B}$.

Adversary $\mathcal{F}_{1}$ randomly chooses $i \leftarrow\{1, \ldots, \kappa(\lambda)\}$ and guesses that $\mathcal{B}$ 's forgery will involve forging an attribute managed by the attribute authority $i$. To answer AddA queries, for all authorities $j \neq i, \mathcal{F}_{1}$ chooses the secret/verification keys for the authority itself. For authority $i$, it sets its verification key to vk it got from its game (and thus it does not know the corresponding secret key). If in the game $\mathcal{B}$ asks a RevealA query on authority $i, \mathcal{F}_{1}$ aborts the game.

To answer AddU queries, $\mathcal{F}_{1}$ chooses the user's key pair itself. To answer AddAtt queries, if the user has attributes managed by authority $i$, it forwards such a query to its Sign oracle; Otherwise, it answers the query itself by using the authorities' secret keys available to it.

To answer Sign queries on (uid, $\mathcal{A}, m, \mathbb{P}$ ), $\mathcal{F}_{1}$ first chooses a fresh key pair (otsvk, otssk) for the one-time signature $\mathcal{O} \mathcal{T} \mathcal{S}$ and encrypts uvk[uid] using $\hat{\mathcal{H}}$ (otsvk) as a tag and generates the rest of the signature $\Sigma$ which it then forwards to $\mathcal{B}$. The rest of $\mathcal{B}$ 's queries are answered normally as in Fig. 1.

Eventually, when $\mathcal{B}$ outputs its forgery, $\mathcal{F}_{1}$ uses the $\mathcal{N} \mathcal{I} \mathcal{Z} \mathcal{K}$ 's extraction key xk to extract the witness and returns the tagged signature on $\mathbf{u v k}\left[\right.$ uid $\left.^{*}\right]$ and the attribute $\alpha^{*}$ if $\mathcal{B}$ 's forgery involved forging a tagged signature. Otherwise, it aborts. $\mathcal{F}_{1}$ also aborts if the forgery does not involve forged attributes managed by authority $i$ that $\mathcal{F}_{1}$ has guessed. The probability of $\mathcal{F}_{1}$ guessing the correct authority is $\frac{1}{\kappa(\lambda)}$.

By the existential unforgeability of the tagged signature scheme, the probability of $\mathcal{B}$ winning is negligible. 
- Adversary $\mathcal{F}_{2}$ : Adversary $\mathcal{F}_{2}$ gets otsvk* from its game and has access to an oracle Sign that it uses to obtain a single one-time signature that verifies w.r.t. otsvk ${ }^{*}$ on a message of its choice. It runs $(\mathrm{crs}, \mathrm{xk}) \leftarrow \mathcal{N} \mathcal{I} \mathcal{Z} \mathcal{K}$.Setup $\left(1^{\lambda}\right)$ and also chooses a key tuple (epk, esvk, esk) for the distributed tag-based encryption scheme $\mathcal{D} \mathcal{T} \mathcal{B}$. It forwards pp $:=\left(1^{\lambda}\right.$, crs, epk, esvk, $\left.\mathcal{H}, \hat{\mathcal{H}}\right)$ and tk $:=$ esk to $\mathcal{B}$.

To answer AddA queries, $\mathcal{F}_{2}$ chooses the authority keys itself. To answer AddU queries, $\mathcal{F}_{2}$ chooses the user's key pair itself. To answer AddAtt queries, $\mathcal{F}_{2}$ uses the corresponding authorities' secret

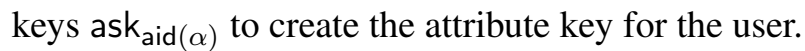

Adversary $\mathcal{F}_{2}$ randomly chooses $i \leftarrow\{1, \ldots, \delta(\lambda)\}$ and guesses that $\mathcal{B}$ 's forgery will involve forging a one-time signature that verifies under otsvk* used in answering the i-th signing query.

When asked for the $\mathrm{j}$-th Sign query on (uid, $\mathcal{A}, m, \mathbb{P}$ ), if $j \neq i, \mathcal{F}_{2}$ chooses a fresh key pair (otsvk, otssk) for the one-time signature scheme and answers the query by itself. If $j=i, \mathcal{F}_{2}$ encrypts uvk[uid] using $\hat{\mathcal{H}}$ (otsvk ${ }^{*}$ ) as a tag to obtain $C_{\text {dtbe }}$ and generates the proof $\pi$. It then forwards $\left(\mathcal{H}(m, \mathbb{P}), \pi, C_{\mathrm{dtbe}}\right.$, otsvk*) as the message to its one-time signature signing oracle to get a one-time signature $\sigma_{\mathrm{ots}} . \mathcal{F}_{2}$ then sends the signature $\Sigma:=\left(\sigma_{\mathrm{ots}}, \pi, C_{\mathrm{dtbe}}\right.$, otsvk $\left.^{*}\right)$ to $\mathcal{B}$. The rest of $\mathcal{B}$ 's queries are answered normally as in Fig. 1.

Eventually, when $\mathcal{B}$ outputs its forgery, $\mathcal{F}_{2}$ aborts if the $\mathcal{B}$ 's forgery did not involve forging a onetime signature that verifies w.r.t otsvk* it got from its game. The probability that $\mathcal{B}$ forges a one-time signature that verifies w.r.t otsvk* is $\frac{1}{\delta(\lambda)}$.

By the strong existential unforgeability of the one-time signature $\mathcal{O} \mathcal{T}, \mathcal{B}$ has a negligible advantage in winning.

This concludes the proof.

Lemma 3. The construction is traceable if the $\mathcal{N I Z K}$ proof system is sound and the tagged signature $\mathcal{T S}$ is existentially unforgeable.

Proof. Since the NIZK proof system $\mathcal{N} \mathcal{I Z K}$ is sound, the adversary has a negligible advantage in succeeding by faking proofs for false statements. We show that if there exists an adversary $\mathcal{B}$ breaking traceability, we can construct an adversary $\mathcal{F}_{1}$ attacking the unforgeability of the tagged signature scheme $\mathcal{T} \mathcal{S}$ such that

$$
\operatorname{Adv}_{\mathcal{D} \mathcal{T} \mathcal{A B S}, \mathcal{B}}^{\text {Trace }}(\lambda) \leq \kappa(\lambda) \cdot \operatorname{Adv}_{\mathcal{T} \mathcal{S}, \mathcal{F}_{1}}^{\text {Unforge }}(\lambda)+\operatorname{Adv}_{\mathcal{N} \mathcal{I} \mathcal{Z} \mathcal{K}, \mathcal{F}_{2}}^{\text {Sound }}(\lambda)
$$

where $\kappa(\lambda)$ is a polynomial in $\lambda$ representing an upper bound on the number of honest attribute authorities $\mathcal{B}$ is allowed to use in the game.

- Adversay $\mathcal{F}_{1}$ : Adversary $\mathcal{F}_{1}$ gets the tagged signature scheme's verification key vk from its game and has access to an oracle Sign that it uses to obtain tagged signatures that verify w.r.t. vk on messages and tags of its choice. Adversary $\mathcal{F}_{1}$ starts by running (crs, xk) $\leftarrow \mathcal{N} \mathcal{I} \mathcal{Z} \mathcal{K}$. Setup $\left(1^{\lambda}\right)$ and choosing a key tuple (epk, esvk, esk) for the distributed tag-based encryption scheme $\mathcal{D} \mathcal{T} \mathcal{B} \mathcal{E}$. It forwards pp $:=\left(1^{\lambda}\right.$, crs, epk, esvk, $\left.\mathcal{H}, \hat{\mathcal{H}}\right)$ and tk $:=$ esk to $\mathcal{B}$.

Adversary $\mathcal{F}_{1}$ randomly chooses $i \leftarrow\{1, \ldots, \kappa(\lambda)\}$ and guesses that $\mathcal{B}$ 's forgery will involve forging an attribute managed by the attribute authority $i$. To answer AddA queries, for all authorities $j \neq i, \mathcal{F}_{1}$ chooses the secret/verification keys for the authority itself. For authority $i$, it sets its verification key to vk it got from its game. If in the game, $\mathcal{B}$ issues RevealA query on authority $i, \mathcal{F}_{1}$ aborts the game. To answer AddU queries, $\mathcal{F}_{1}$ chooses the user's key pair itself. Whenever asked AddAtt queries, if the user has attributes managed by authority $i$, it forwards such a query to its Sign oracle; Otherwise, it answers the query itself.

To answer Sign queries on (uid, $\mathcal{A}, m, \mathbb{P}$ ), $\mathcal{F}_{1}$ first chooses a fresh key pair (otsvk, otssk) for the onetime signature $\mathcal{O} \mathcal{T S}$ and encrypts uvk[uid] using $\hat{\mathcal{H}}$ (otsvk) as a tag and generates the rest of the 
signature by itself. $\mathcal{F}_{1}$ forwards the signature $\Sigma$ to $\mathcal{B}$. The rest of $\mathcal{B}$ 's queries are answered normally as in Fig. 1.

Eventually, when $\mathcal{B}$ outputs its forgery, $\mathcal{F}_{1}$ uses the $\mathcal{N} \mathcal{I} \mathcal{Z} \mathcal{K}$ 's extraction key xk to extract the witness and returns the tagged signature on the $\mathbf{u v k}\left[\mathrm{uid}^{*}\right]$ and the attribute $\alpha^{*}$ if $\mathcal{B}$ 's forgery involved forging a tagged signature that verifies w.r.t. vk it got from its game. Otherwise, it aborts. The probability that $\mathcal{F}_{1}$ guesses the correct authority is $\frac{1}{\kappa(\lambda)}$.

By the existential unforgeability of the tagged signature, the probability of $\mathcal{B}$ winning is negligible.

This concludes the proof

Lemma 4. If $\mathcal{N} \mathcal{I Z K}$ is zero-knowledge, the distributed tag-based encryption scheme $\mathcal{D} \mathcal{T} B \mathcal{B}$ is selectivetag weakly IND-CCA secure, the one-time signature $\mathcal{O} \mathcal{T} \mathcal{S}$ is strongly existentially unforgeable, and the hash functions $\hat{\mathcal{H}}$ and $\mathcal{H}$ are collision-resistant then the construction is fully anonymous (against full-key exposure).

Proof. We show that if there exists an adversary $\mathcal{B}$ breaking anonymity, we can construct adversaries: $\mathcal{F}_{1}$ against the collision-resistance of the hash function $\hat{\mathcal{H}}, \mathcal{F}_{2}$ against the strong unforgeability of the one-time signature $\mathcal{O} \mathcal{T}, \mathcal{F}_{3}$ against the collision-resistance of the hash function $\mathcal{H}, \mathcal{F}_{4}$ against the NIZK property of the proof system $\mathcal{N} \mathcal{I Z K}$, and $\mathcal{F}_{5}$ against the selective-tag weakly IND-CCA security of the distributed tag-based encryption scheme $\mathcal{D} \mathcal{T} \mathcal{B E}$.

By the collision-resistance of $\hat{\mathcal{H}}, \mathcal{B}$ has a negligible probability in finding otsvk' s.t. $\hat{\mathcal{H}}\left(\right.$ otsvk $\left.^{\prime}\right)$ collides with the tag $\hat{\mathcal{H}}\left(\right.$ otsvk $\left.^{*}\right)$ we use for the challenge signature. If this is not the case, we can use $\mathcal{B}$ to construct an adversary $\mathcal{F}_{1}$ that breaks the collision-resistance of $\hat{\mathcal{H}}$.

The strong existential unforgeability of $\mathcal{O} \mathcal{T S}$ ensures that $\mathcal{B}$ has a negligible probability in forging a one-time signature under otsvk* we use in the challenge signature. If this is not the case, we can construct an adversary $\mathcal{F}_{2}$ that wins the strong unforgeability game of $\mathcal{O T S}$.

By the collision-resistance of $\mathcal{H}, \mathcal{B}$ has a negligible probability in finding pairs $\left(m^{*}, \mathbb{P}^{*}\right) \neq(m, \mathbb{P})$ s.t. $\mathcal{H}\left(m^{*}, \mathbb{P}^{*}\right)=\mathcal{H}(m, \mathbb{P})$. If this is not the case, we can use $\mathcal{B}$ to construct an adversary $\mathcal{F}_{3}$ which breaks the collision-resistance of the hash function $\mathcal{H}$. Thus, from now on we assume that there are no hash collisions.

We instantiate $\mathcal{N} \mathcal{I Z K}$ in the simulation setting which is, by the security of $\mathcal{N} \mathcal{I Z K}$, is indistinguishable from the soundness setting. The proof $\pi$ is thus now zero-knowledge and hence does not reveal any information about the witness.

We now proceed to construct an adversary $\mathcal{F}_{5}$ against the selective-tag weakly IND-CCA security of $\mathcal{D} \mathcal{T} \mathcal{B}$ using adversary $\mathcal{B}$. Adversary $\mathcal{F}_{5}$ runs the Setup algorithm where it starts by randomly choosing a key pair (otsvk*, otssk*) for $\mathcal{O} \mathcal{T} S$ that it will use when producing the challenge signature. We needed to choose the key pair beforehand as the distributed tag-based encryption scheme is only selective-tag secure and hence the challenger in the ST-wIND-CCA game needs to know the challenge tag before sending epk and esvk. $\mathcal{F}_{5}$ sends $\hat{\mathcal{H}}$ (otsvk*) to its challenger and gets back epk and esvk. In its game, $\mathcal{F}_{5}$ has access to a decryption oracle Dec which it can query on any ciphertext under any tag different from $\hat{\mathcal{H}}\left(\right.$ otsvk $\left.^{*}\right) . \mathcal{F}_{5}$ chooses crs as a simulation reference string and forwards $\mathrm{pp}:=\left(1^{\lambda}\right.$, crs, epk, esvk, $\hat{\mathcal{H}}, \mathcal{H})$ to $\mathcal{B}$.

To answer AddU queries, $\mathcal{F}_{5}$ chooses the secret/verification keys of the user itself. To answer AddA queries, $\mathcal{F}_{5}$ chooses the secret/verification keys for the authorities itself. Thus, $\mathcal{F}_{5}$ can answer any AddAtt queries itself.

To answer the challenge query $\mathrm{CH}_{b}\left(\left(\right.\right.$ uid $\left._{0}, \mathcal{A}_{0}\right),\left(\right.$ uid $\left.\left._{1}, \mathcal{A}_{1}\right), m, \mathbb{P}\right), \mathcal{F}_{5}$ sends (uvk $[$ uid 0$], \mathbf{u v k}\left[\right.$ uid $\left.\left._{1}\right]\right)$ as its challenge in its ST-wIND-CCA game and gets a ciphertext under the tag $\hat{\mathcal{H}}$ (otsvk*) of either the plaintext $\mathbf{u v k}\left[\right.$ uid $\left._{0}\right]$ or $\mathbf{u v k}\left[\right.$ uid $\left._{1}\right]$ which it needs to distinguish. $\mathcal{F}_{5}$ can now construct the rest of the challenge signature by simulating the proof $\pi$ and signing the whole thing with otssk* to obtain $\sigma_{\text {ots. }}$.

To answer Trace queries, $\mathcal{F}_{5}$ just uses its decryption oracle to get the decryption shares of $C_{\text {dtbe }}$. Note that since we have chosen the challenge tag otsvk* uniformly at random and since we already eliminated 
any case where any signature sent to Trace uses the same tag as that we used for the challenge signature, such a query will be accepted by $\mathcal{F}_{5}$ 's decryption oracle because the tag is different from the tag used in the challenge ciphertext. The rest of $\mathcal{B}$ 's queries are answered as in Fig. 1.

Eventually, when $\mathcal{B}$ halts, $\mathcal{F}_{5}$ outputs whatever $\mathcal{B}$ outputs. By the ST-wIND-CCA property of the distributed tag-based encryption scheme, $\mathcal{B}$ has a negligible probability in winning.

This concludes the proof.

Lemma 5. The construction satisfies tracing soundness if $\mathcal{D} \mathcal{T} \mathcal{B}$ satisfies decryption consistency.

Proof. We show that if there exists an adversary $\mathcal{B}$ that wins the tracing soundness game then we can build an adversary $\mathcal{F}$ that breaks the decryption consistency requirement of the distributed tag-based encryption scheme $\mathcal{D} \mathcal{T} B \mathcal{E}$ such that

$$
\operatorname{Adv}_{\mathcal{D} \mathcal{T} \mathcal{A B S}, \mathcal{B}}^{\mathrm{TS}}(\lambda) \leq \operatorname{Adv}_{\mathcal{D} \mathcal{T} \mathcal{B} \mathcal{F}, \mathcal{F}, 1}^{\mathrm{DEC}-C O N}(\lambda)
$$

Adversary $\mathcal{F}$ gets (epk, esvk, esk) from its game and runs the rest of the Setup algorithm normally. $\mathcal{F}$ forwards pp $:=\left(1^{\lambda}\right.$, crs, epk, esvk, $\left.\mathcal{H}, \hat{\mathcal{H}}\right)$ and tk $:=$ esk to $\mathcal{B}$. All $\mathcal{B}$ 's queries are answered normally as in Fig. 1. Eventually, when $\mathcal{B}$ halts, $\mathcal{F}$ outputs $\nu$ and $\nu^{\prime}$ returned by $\mathcal{B}$ in its game.

By the decryption consistency property of the distributed tag-based encryption scheme $\mathcal{D} \mathcal{T} \mathcal{B}$, this only happens with a negligible probability.

This concludes the proof. 